\title{
Spinning Strings and Integrable Spin Chains in the AdS/CFT Correspondence
}

\author{
Jan Plefka \\ Max Planck Institute for Gravitational Physics \\ Am Mühlenberg 1, 14476 Potsdam, Germany \\ email: jan.plefka@aei.mpg.de \\ http://www.aei.mpg.de/ plefka/ \\ Accepted on 20 October 2005 \\ Published on 30 November 2005 \\ Living Reviews in Relativity \\ Published by the \\ Max Planck Institute for Gravitational Physics \\ (Albert Einstein Institute) \\ Am Mühlenberg 1, 14424 Golm, Germany \\ ISSN $1433-8351$
}

\begin{abstract}
In this introductory review we discuss dynamical tests of the $A d S_{5} \times S^{5}$ string $/ \mathcal{N}=4$ Super Yang-Mills duality. After a brief introduction to AdS/CFT, we argue that semiclassical string energies yield information on the quantum spectrum of the string in the limit of large angular momenta on the $S^{5}$. The energies of the folded and circular spinning string solutions rotating on a $S^{3}$ within the $S^{5}$ are derived, which yield all-loop predictions for the dual gauge theory scaling dimensions. These follow from the eigenvalues of the dilatation operator of $\mathcal{N}=4$ Super Yang-Mills in a minimal SU(2) subsector, and we display its reformulation in terms of a Heisenberg $s=1 / 2$ spin chain along with the coordinate Bethe ansatz for its explicit diagonalization. In order to make contact to the spinning string energies, we then study the thermodynamic limit of the one-loop gauge theory Bethe equations and demonstrate the matching with the folded and closed string result at this loop order. Finally, the known gauge theory results at higher-loop orders are reviewed and the associated long-range spin chain Bethe ansatz is introduced, leading to an asymptotic all-loop conjecture for the gauge theory Bethe equations. This uncovers discrepancies at the three-loop order between gauge theory scaling dimensions and string theory energies and the implications of this are discussed. Along the way, we comment on further developments and generalizations of the subject and point to the relevant literature.
\end{abstract}

(c) Max Planck Society and the authors.

Further information on copyright is given at http://relativity.livingreviews.org/About/copyright.html For permission to reproduce the article please contact livrev@aei.mpg.de. 


\section{How to cite this article}

Owing to the fact that a Living Reviews article can evolve over time, we recommend to cite the article as follows:

\section{Jan Plefka,}

"Spinning Strings and Integrable Spin Chains in the AdS/CFT Correspondence",

Living Rev. Relativity, 8, (2005), 9. [Online Article]: cited [<date $>$ ],

http://www.livingreviews.org/lrr-2005-9

The date given as $<$ date $>$ then uniquely identifies the version of the article you are referring to.

\section{Article Revisions}

Living Reviews supports two different ways to keep its articles up-to-date:

Fast-track revision A fast-track revision provides the author with the opportunity to add short notices of current research results, trends and developments, or important publications to the article. A fast-track revision is refereed by the responsible subject editor. If an article has undergone a fast-track revision, a summary of changes will be listed here.

Major update A major update will include substantial changes and additions and is subject to full external refereeing. It is published with a new publication number.

For detailed documentation of an article's evolution, please refer always to the history document of the article's online version at http: //www. livingreviews.org/lrr-2005-9. 


\section{Contents}

1 Introduction $\quad 5$

2 The Setup $\quad 8$

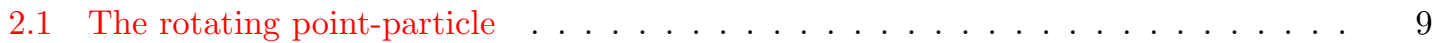

$2.2 \mathcal{N}=4$ Super Yang-Mills . . . . . . . . . . . . . . . . . . . . 10

3 Spinning String Solutions 12

3.1 The special case: $\omega_{1}=\omega_{2} \ldots \ldots \ldots \ldots \ldots \ldots \ldots$. . . . . . . . . . . . . . . . . . .

3.2 The folded string: $q \leq 1 \ldots \ldots \ldots \ldots$

3.3 The circular string: $q>1 \ldots \ldots \ldots \ldots$

3.4 Further Developments . . . . . . . . . . . . . . . . . 15

4 The Dual Gauge Theory Side $\quad 18$

4.1 The coordinate Bethe ansatz . . . . . . . . . . . . . . . . . 19

4.2 The thermodynamic limit of the spin chain . . . . . . . . . . . . . . . . . . . 22

4.3 Higher Loops in the $\mathrm{SU}(2)$ sector and discrepancies . . . . . . . . . . . . . . . . . . . . . 24

4.4 Further developments . . . . . . . . . . . . . . . . . . . 27

5 Acknowledgments $\quad 29$

$\begin{array}{lr}\text { References } & 39\end{array}$ 



\section{Introduction}

String theory was initially discovered in an attempt to describe the physics of the strong interactions prior to the advent of gauge field theories and QCD. Today, it has matured to a very promising candidate for a unified quantum theory of gravity and all the other forces of nature. In this interpretation, gauge fields arise as the low energy excitations of fundamental open strings and are therefore derived, non-fundamental objects, just as the theory of gravity itself. Ironically though, advances in our understanding of non-perturbative string theory and of D-branes have led to a resurrection of gauge fields as fundamental objects. Namely, it is now generally believed that string theory in suitable space-time backgrounds can have a dual, holographic description in terms of gauge field theories and thus the question, which of the two is the fundamental one, becomes redundant. This belief builds on a remarkable proposal due to Maldacena [86] known as the Anti-de-Sitter/Conformal Field Theory (AdS/CFT) correspondence (for reviews see [1, 52]).

The initial idea of a string/gauge duality is due to 't Hooft [115], who realized that the perturbative expansion of $\mathrm{SU}(N)$ gauge field theory in the large $N$ limit can be reinterpreted as a genus expansion of discretized two-dimensional surfaces built from the field theory Feynman diagrams. Here $1 / N$ counts the genus of the Feynman diagram, while the 't Hooft coupling $\lambda:=g_{\mathrm{YM}}^{2} N$ (with $g_{\mathrm{YM}}$ denoting the gauge theory coupling constant) enumerates quantum loops. The genus expansion of the free energy $F$ of a SU(N) gauge theory in the 't Hooft limit $(N \rightarrow \infty$ with $\lambda$ fixed), for example, takes the pictorial form

$$
F=N^{2} \quad+1
$$

with suitable coefficients $c_{g, l}$ denoting the contributions at genus $g$ and loop order $l$. Obviously, this $1 / N$ expansion resembles the perturbative expansion of a string theory in the string coupling constant $g_{S}$.

The AdS/CFT correspondence is the first concrete realization of this idea for four-dimensional gauge theories. In its purest form - which shall also be the setting we will be interested in - it identifies the "fundamental" type IIB superstring in a ten-dimensional anti-de-Sitter cross sphere $\left(A d S_{5} \times S^{5}\right)$ space-time background with the maximally supersymmetric Yang-Mills theory with gauge group $\mathrm{SU}(N)(\mathcal{N}=4 \mathrm{SYM})$ in four dimensions. The $\mathcal{N}=4$ Super Yang-Mills model is a quantum conformal field theory, as its $\beta$-function vanishes exactly. The string model is controlled by two parameters: the string coupling constant $g_{S}$ and the "effective" string tension $R^{2} / \alpha^{\prime}$, where $R$ is the common radius of the $A d S_{5}$ and $S_{5}$ geometries. The gauge theory, on the other hand, is parameterized by the rank $N$ of the gauge group and the coupling constant $g_{\mathrm{YM}}$, or equivalently, the 't Hooft coupling $\lambda:=g_{\mathrm{YM}}^{2} N$. According to the AdS/CFT proposal, these two sets of parameters are to be identified as

$$
\frac{4 \pi \lambda}{N}=g_{S} \quad \sqrt{\lambda}=\frac{R^{2}}{\alpha^{\prime}} .
$$

We see that in the AdS/CFT proposal the string coupling constant is not simply given by $1 / N$, but comes with a linear factor in $\lambda$. This, however, does not alter the genus expansion and its interpretation in form of string worldsheets.

The equations (2) relate the coupling constants, but there is also a dictionary between the excitations of the two theories. The correspondence identifies the energy eigenstates of the $A d S_{5} \times S^{5}$ string, which we denote schematically as $\left|\mathcal{O}_{A}\right\rangle$ - with $A$ being a multi-index - with (suitable) composite gauge theory operators of the form $\mathcal{O}_{A}=\operatorname{Tr}\left(\phi_{i_{1}} \phi_{i_{2}} \ldots \phi_{i_{n}}\right)$, where $\left(\phi_{i}\right)_{a b}$ are the elementary fields of $\mathcal{N}=4 \mathrm{SYM}$ (and their covariant derivatives) in the adjoint representation of $\mathrm{SU}(N)$, i.e. $N \times N$ hermitian matrices. The energy eigenvalue $E$ of a string state, with respect to time in global coordinates, is conjectured to be equal to the scaling dimension $\Delta$ of the dual gauge theory 
operator, which in turn is determined from the two point function of the conformal field theory ${ }^{1}$

$$
\left\langle\mathcal{O}_{A}(x) \mathcal{O}_{B}(y)\right\rangle=\frac{M \delta_{A, B}}{(x-y)^{2 \Delta_{A}\left(\lambda, \frac{1}{N}\right)}} \quad \Leftrightarrow \quad \mathcal{H}_{\text {String }}\left|\mathcal{O}_{A}\right\rangle=E_{A}\left(\frac{R^{2}}{\alpha^{\prime}}, g_{S}\right)\left|\mathcal{O}_{A}\right\rangle
$$

with $\Delta(\lambda, 1 / N) \stackrel{!}{=} E\left(R^{2} / \alpha^{\prime}, g_{S}\right)$.

A zeroth order test of the conjecture is then the agreement of the underlying symmetry supergroup $\operatorname{PSU}(2,2 \mid 4)$ of the two theories, which furnishes the representations under which $\mathcal{O}_{A}(x)$ and $\left|\mathcal{O}_{A}\right\rangle$ transform. This then yields a hint on how one could set up an explicit string state/gauge operator dictionary.

Clearly, there is little hope of determining either the all genus (all orders in $g_{S}$ ) string spectrum, or the complete $1 / N$ dependence of the gauge theory scaling dimensions $\Delta$. But the identification of the planar gauge theory with the free $\left(g_{S}=0\right)$ string seems feasible and fascinating: Free $A d S_{5} \times S^{5}$ string theory should give the exact all-loop gauge theory scaling dimensions in the large $N$ limit! Unfortunately though, our knowledge of the string spectrum in curved backgrounds, even in such a highly symmetric one as $A d S_{5} \times S^{5}$, remains scarce. Therefore, until very recently, investigations on the string side of the correspondence were limited to the domain of the low energy effective field theory description of $A d S_{5} \times S^{5}$ strings in terms of type IIB supergravity. This, however, is necessarily limited to weakly curved geometries in string units, i.e. to the domain of $\sqrt{\lambda} \gg 1$ by virtue of (2). On the gauge theory side, one has control only in the perturbative regime where $\lambda \ll 1$, which is perfectly incompatible with the accessible supergravity regime $\sqrt{\lambda} \gg 1$. Hence, one is facing a strong/weak coupling duality, in which strongly coupled gauge fields are described by classical supergravity, and weakly coupled gauge fields correspond to strings propagating in a highly curved background geometry. This insight is certainly fascinating, but at the same time strongly hinders any dynamical tests (or even a proof) of the AdS/CFT conjecture in regimes that are not protected by the large amount of symmetry in the problem.

This situation has profoundly changed since 2002 by performing studies of the correspondence in novel limits where quantum numbers (such as spins or angular momenta in the geometric $A d S_{5} \times S^{5}$ language) become large in a correlated fashion as $N \rightarrow \infty$. This was initiated in the work of Berenstein, Maldacena and Nastase [33], who considered the quantum fluctuation expansion of the string around a degenerated point-like configuration, corresponding to a particle rotating with a large angular momentum $J$ on a great circle of the $S^{5}$ space. In the limit of $J \rightarrow \infty$ with $J^{2} / N$ held fixed (the "BMN limit"), the geometry seen by the fast moving particle is a gravitational plane-wave, which allows for an exact quantization of the free string in the lightcone-gauge $[88,90]$. The resulting string spectrum leads to a formidable prediction for the all-loop scaling dimensions of the dual gauge theory operators in the corresponding limit, i.e. the famous formula $\Delta_{n}=J+2 \sqrt{1+\lambda n^{2} / J^{2}}$ for the simplest two string oscillator mode excitation. The key point here is the emergence of the effective gauge theory loop counting parameter $\lambda / J^{2}$ in the BMN limit. By now, these scaling dimensions have been firmly reproduced up to the three-loop order in gauge theory $[21,12,53]$. This has also led to important structural information for higher (or all-loop) attempts in gauge theory, which maximally employ the uncovered integrable structures to be discussed below. Moreover, the plane wave string theory $/ \mathcal{N}=4$ SYM duality could be extended to the interacting string $\left(g_{S} \neq 0\right)$ respectively non-planar gauge theory regime providing us with the most concrete realization of a string/gauge duality to date (for reviews see [94, 97, 104, 103]).

In this review we shall discuss developments beyond the BMN plane-wave correspondence, which employ more general sectors of large quantum numbers in the AdS/CFT duality. The key point from the string perspective is that such a limit can make the semiclassical (in the planewave case) or even classical (in the "spinning string" case) computation of the string energies also quantum exact [61,62], i.e. higher $\sigma$-model loops are suppressed by inverse powers of the total

\footnotetext{
${ }^{1}$ For simplicity we take $\mathcal{O}_{A}(x)$ to be a scalar operator here.
} 
angular momentum $J$ on the five sphere ${ }^{2}$. These considerations on the string side then (arguably) yield all-loop predictions for the dual gauge theory. Additionally, the perturbative gauge theoretic studies at the first few orders in $\lambda$ led to the discovery that the spectrum of scaling dimensions of the planar gauge theory is identical to that of an integrable long-range spin chain [92, 21, 24]. Consistently, the $A d S_{5} \times S^{5}$ string is a classically integrable model [31], which has been heavily exploited in the construction of spinning string solutions.

This review aims at a more elementary introduction to this very active area of research, which in principle holds the promise of finding the exact quantum spectrum of the $A d S_{5} \times S^{5}$ string or equivalently the all-loop scaling dimensions of planar $\mathcal{N}=4$ Super Yang-Mills. It is intended as a first guide to the field for students and interested "newcomers" and points to the relevant literature for deeper studies. We will discuss the simplest solutions of the $A d S_{5} \times S^{5}$ string corresponding to folded and circular string configurations propagating in a $\mathbb{R} \times S^{3}$ subspace, with the $S^{3}$ lying within the $S^{5}$. On the gauge theory side we will motivate the emergence of the spin chain picture at the leading one-loop order and discuss the emerging Heisenberg $\mathrm{XXX}_{1 / 2}$ model and its diagonalization using the coordinate Bethe ansatz technique. This then enables us to perform a comparison between the classical string predictions in the limit of large angular momenta and the dual thermodynamic limit of the spin chain spectrum. Finally, we turn to higher-loop calculations in the gauge-theory and discuss conjectures for the all-loop form of the Bethe equations, giving rise to a long-range interacting spin chain. Comparison with the obtained string results uncovers a discrepancy from loop order three onwards and the interpretation of this result is also discussed.

A number of more detailed reviews on spinning strings, integrability and spin chains in the AdS/CFT correspondence already exist: Tseytlin's review [117] mostly focuses on the string side of the correspondence, whereas Beisert's Physics Report [13] concentrates primarily on the gauge side. See also Tseytlin's second review [116], on the so-called coherent-state effective action approach, which we will not discuss in this review. Recommended is also the shorter review by Zarembo [119] on the $\mathrm{SU}(2)$ respectively $\mathbb{R} \times S^{3}$ subsector, discussing the integrable structure appearing on the classical string - not covered in this review. For a detailed account of the near plane-wave superstring, its quantum spectroscopy and integrability structures see Swanson's thesis [114].

\footnotetext{
${ }^{2}$ This suppression only occurs if at least one angular momentum on the five sphere becomes large. If this is not the case, e.g. for a spinning string purely on the $A d S_{5}$ [69], quantum corrections are not suppressed by inverse powers of the spin on $A d S_{5}$ [60].
} 


\section{The Setup}

With the embedding coordinates $X^{m}(\tau, \sigma)$ and $Y^{m}(\tau, \sigma)$ the Polyakov action of the $A d S_{5} \times S^{5}$ string in conformal gauge $\left(\eta_{a b}=\operatorname{diag}(-1,1)\right)$ takes the form $(m, n=1,2,3,4,5)$

$$
I=-\frac{\sqrt{\lambda}}{4 \pi} \int d \tau d \sigma\left[G_{m n}^{\left(A d S_{5}\right)} \partial_{a} X^{m} \partial^{a} X^{n}+G_{m n}^{\left(S^{5}\right)} \partial_{a} Y^{m} \partial^{a} Y^{n}\right]+\text { fermions }
$$

where we have suppressed the fermionic terms in the action, as they will not be relevant in our discussion of classical solutions (the full fermionic action is stated in [89, 100]). A natural choice of coordinates for the $A d S_{5} \times S^{5}$ space ("global coordinates") is ${ }^{3}$

$$
\begin{aligned}
d s_{A d S_{5}}^{2} & =d \rho^{2}-\cosh ^{2} \rho d t^{2}+\sinh ^{2} \rho\left(d \bar{\psi}^{2}+\cos ^{2} \bar{\psi} d \varphi_{1}{ }^{2}+\sin ^{2} \bar{\psi} d \varphi_{2}{ }^{2}\right) \\
d s_{S^{5}}^{2} & =d \gamma^{2}+\cos ^{2} \gamma d \phi_{3}{ }^{2}+\sin ^{2} \gamma\left(d \psi^{2}+\cos ^{2} \psi d \phi_{1}{ }^{2}+\sin ^{2} \psi d \phi_{2}{ }^{2}\right)
\end{aligned}
$$

Moreover, we have directly written the string action with the help of the effective string tension $\sqrt{\lambda}=R^{2} / \alpha^{\prime}$ of (2). It is helpful to picture the $A d S_{5}$ space-time as a bulk cylinder with a fourdimensional boundary of the form $\mathbb{R} \times S^{3}$ (see Figure 3 ).

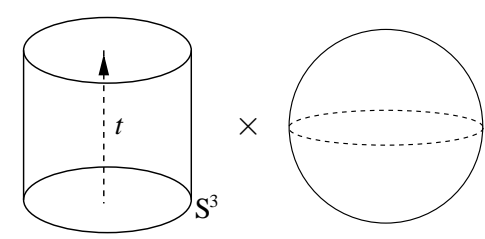

Figure 1: Sketch of the $A d S_{5}$ (bulk cylinder with boundary $R \times S^{3}$ ) space-time and the $S^{5}$ (sphere) space.

A consequence of the conformal gauge choice are the Virasoro constraints, which take the form

$$
0 \stackrel{!}{=} \dot{X}^{m} X_{m}^{\prime}+\dot{Y}^{p} Y_{p}^{\prime} \quad 0 \stackrel{!}{=} \dot{X}^{m} \dot{X}_{m}+\dot{Y}^{p} \dot{Y}_{p}+X^{m \prime} X_{m}^{\prime}+Y^{p^{\prime}} Y_{p}^{\prime},
$$

where the dot refers to $\partial_{\tau}$ and the prime to $\partial_{\sigma}$ derivatives. Of course the contractions in the above are to be performed with the metrics of (5).

As mentioned in the introduction, it is presently unknown how to perform an exact quantization of this model. It is, however, possible to perform a quantum fluctuation expansion in $1 / \sqrt{\lambda}$. For this one expands around a classical solution of (4) and integrates out the fluctuations in the pathintegral loop order by order. This is the route we will follow. Of course, in doing so, we will only have a patch-wise access to the full spectrum of the theory, with each patch given by the solution expanded around.

\footnotetext{
${ }^{3}$ This metric arises from a parameterization of the five sphere $x_{1}^{2}+x_{2}^{2}+x_{3}^{2}+\cdots+x_{6}^{2}=1$ and anti-de-Sitter space $-y_{-1}^{2}-y_{0}^{2}+y_{1}^{2}+y_{2}^{2}+\cdots y_{4}^{2}=-1$ through

$$
\begin{aligned}
& x_{1}+i x_{2}=\sin \gamma \cos \psi e^{i \phi_{1}}, \quad x_{3}+i x_{4}=\sin \gamma \sin \psi e^{i \phi_{2}}, \quad x_{5}+i x_{6}=\cos \gamma e^{i \phi_{3}} ; \\
& y_{1}+i y_{2}=\sinh \rho \cos \bar{\psi} e^{i \varphi_{1}}, \quad y_{3}+i y_{4}=\sinh \rho \sin \bar{\psi} e^{i \varphi_{2}}, \quad y_{-1}+i y_{0}=\cosh \rho e^{i t} .
\end{aligned}
$$

The embedding coordinates $X^{m}(\tau, \sigma)$ and $Y^{m}(\tau, \sigma)$ in $(4)$ are hence given by $X^{m}=\left(\rho, \bar{\psi}, \varphi_{1}, \varphi_{2}, t\right)$ and $Y^{m}=$ $\left(\gamma, \psi, \phi_{1}, \phi_{2}, \phi_{3}\right)$.

Living Reviews in Relativity

http://www. livingreviews . org//rr-2005-9 


\subsection{The rotating point-particle}

It is instructive to sketch this procedure by considering the perhaps simplest solution to the equations of motion of (4): the rotating point-particle on $S^{5}$, which is a degenerated string configuration.

$$
t=\kappa \tau, \quad \rho=0, \quad \gamma=\frac{\pi}{2}, \quad \phi_{1}=\kappa \tau, \quad \phi_{2}=\phi_{3}=\psi=0 .
$$

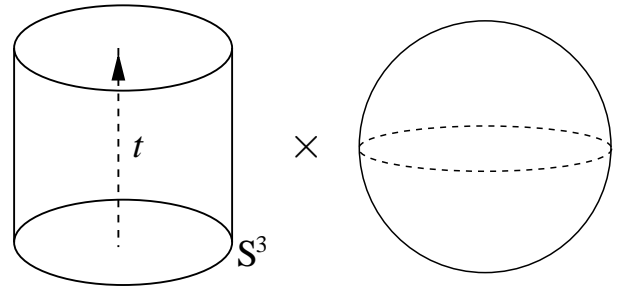

One easily shows that this configurations satisfies the equations of motion and the Virasoro constraints.

A glance at Equations $(4,5)$ reveals that the cyclic coordinates of the action $I=\int d t L$ are $\left(t, \varphi_{1}, \varphi_{2} ; \phi_{1}, \phi_{2}, \phi_{3}\right)$ leading to the conserved charges $\left(E, S_{1}, S_{2} ; J_{1}, J_{2}, J_{3}\right)$, corresponding to the energy $E$ and two spins $\left(S_{1}, S_{2}\right)$ on $A d S_{5}$ as well as the three angular momenta $\left(J_{1}, J_{2}, J_{3}\right)$ on the five sphere respectively. The energy $E$ and the first angular momentum $J_{1}$ are the only non vanishing conserved quantities of the above point-particle configuration and take the values (we also spell out $J_{2}$ for later use)

$$
\begin{aligned}
E & :=\frac{\partial L}{\partial \dot{t}}=\sqrt{\lambda} \int_{0}^{2 \pi} \frac{d \sigma}{2 \pi} \cosh ^{2} \rho \dot{t}=\sqrt{\lambda} \kappa, \\
J_{1} & :=-\frac{\partial L}{\partial \dot{\phi}_{1}}=\sqrt{\lambda} \int_{0}^{2 \pi} \frac{d \sigma}{2 \pi} \sin ^{2} \gamma \cos ^{2} \psi \dot{\phi}_{1}=\sqrt{\lambda} \kappa, \\
J_{2} & :=-\frac{\partial L}{\partial \dot{\phi}_{2}}=\sqrt{\lambda} \int_{0}^{2 \pi} \frac{d \sigma}{2 \pi} \sin ^{2} \gamma \sin ^{2} \psi \dot{\phi}_{2}=0, \\
J & :=J_{1}+J_{2} .
\end{aligned}
$$

Hence classically $E=J$. One may now consider quantum fluctuations around this solution, i.e. $X^{\mu}=X_{\text {solution }}^{\mu}(\tau)+\frac{1}{\lambda^{1 / 4}} x^{\mu}(\tau, \sigma)$, and integrate out the quantum field $x^{\mu}(\tau, \sigma)$ in a perturbative fashion. This will result in "quantum" corrections to the classical energy $E_{0}$ in terms of an expansion in $1 / \sqrt{\lambda}$

$$
E=\sqrt{\lambda} \kappa+E_{2}(\kappa)+\frac{1}{\sqrt{\lambda}} E_{4}(\kappa)+\cdots
$$

They key idea of Berenstein, Maldacena and Nastase [33] was to consider the limit $J \rightarrow \infty$ with the parameter $\kappa=J / \sqrt{\lambda}$ held fixed. This limit of a large quantum number suppresses all the higher loop contributions beyond one-loop, i.e.

$$
E-J=E_{2}(\kappa)+\frac{1}{\sqrt{J}} \tilde{E}_{4}(\kappa)+\cdots \stackrel{J \rightarrow \infty}{\longrightarrow} E_{2}(\kappa) .
$$

Hence, the quadratic approximation becomes exact! This quadratic fluctuation action (including the fermions) is nothing but the IIB superstring in a plane wave background [88], which arises from the $A d S_{5} \times S^{5}$ geometry through a so-called Penrose limit (see [38, 39] for this construction). The quantization of this string model is straightforward in the light-cone gauge [88, 90] and leads to a free, massive two-dimensional theory for the transverse degrees of freedom $(i=1, \ldots, 8)$

$$
I_{2}=\int d \tau d \sigma\left(\frac{1}{2} \partial_{a} x^{i} \partial_{a} x^{i}-\frac{\kappa^{2}}{2} x^{i} x^{i}+\text { fermions }\right)
$$


with a compact expression for the spectrum

$$
E_{2}=\frac{1}{\sqrt{\lambda^{\prime}}} \sum_{n=-\infty}^{\infty} \sqrt{1+\lambda^{\prime} n^{2}} \widehat{N}_{n} \quad \lambda^{\prime}:=\frac{1}{\sqrt{\kappa}}=\frac{\lambda}{J^{2}}
$$

where $\widehat{N}_{n}:=\alpha_{n}^{\dagger i} \alpha_{n}^{i}$ is the excitation number operator for transverse string excitations $\alpha_{n}^{\dagger i}|0\rangle$ with $\left[\alpha_{m}^{i}, \alpha_{n}^{\dagger j}\right]=\delta_{n m} \delta_{i j}$. The Virasoro constraints (6) reduce to the level matching condition $\sum_{n} n \widehat{N}_{n}=0$ known from string theory in flat Minkowski space-time. Hence, the first stringy excitation is $\alpha_{n}^{\dagger} \alpha_{-n}^{\dagger}|0\rangle$ with $\sqrt{\lambda^{\prime}} E_{2}=2 \sqrt{1+\lambda^{\prime} n^{2}}$. For a more detailed treatment of the plane wave superstring see [94, 97, 104, 103].

\section{$2.2 \mathcal{N}=4$ Super Yang-Mills}

The conjectured dual gauge theory of the $A d S_{5} \times S^{5}$ superstring is the maximally supersymmetric $(\mathcal{N}=4)$ Yang-Mills theory in four dimensions [66, 42]. Its field content is given by a gluon field $A_{\mu}(x)$, six scalars $\phi_{i}(x)(i=1, \ldots, 6)$ as well as 4 Majorana gluinos, which we choose to write as a 16 component 10d Majorana-Weyl spinor $\chi_{\alpha}(x)(\alpha=1, \ldots 16)$. All fields are in the adjoint representation of $\mathrm{SU}(N)$. The action of $\mathcal{N}=4$ Super Yang-Mills is uniquely determined by two parameters, the coupling constant $g_{\mathrm{YM}}$ and the rank of the gauge group $\mathrm{SU}(N)$

$$
S=\frac{2}{g_{\mathrm{YM}}^{2}} \int d^{4} x \operatorname{Tr}\left\{\frac{1}{4}\left(F_{\mu \nu}\right)^{2}+\frac{1}{2}\left(D_{\mu} \phi_{i}\right)^{2}-\frac{1}{4}\left[\phi_{i}, \phi_{j}\right]\left[\phi_{i}, \phi_{j}\right]+\frac{1}{2} \bar{\chi} \not D \chi-\frac{i}{2} \bar{\chi} \Gamma_{i}\left[\phi_{i}, \chi\right]\right\}
$$

with the covariant derivative $D_{\mu}=\partial_{\mu}-i\left[A_{\mu},\right]$. Furthermore, $\left(\Gamma_{\mu}, \Gamma_{i}\right)$ are the ten-dimensional Dirac matrices.

Due to the large amount of supersymmetry present, the conformal invariance of the classical field theory survives the quantization procedure: The coupling constant $g_{\mathrm{YM}}$ is not renormalized and its $\beta$-function vanishes to all orders in perturbation theory [108, 72, 41]. This is why one often refers to $\mathcal{N}=4$ Super Yang-Mills as a "finite" quantum field theory. Nevertheless, composite gauge invariant operators, i.e. traces of products of fundamental fields and their covariant derivatives at the same space-point, e.g. $\mathcal{O}_{i_{1} \ldots i_{k}}(x)=\operatorname{Tr}\left[\phi_{i_{1}}(x) \phi_{i_{2}}(x) \ldots \phi_{i_{k}}(x)\right]$, are renormalized and acquire anomalous dimensions. These may be read off from the two point functions (stated here for the case of scalar operators)

$$
\left\langle\mathcal{O}_{A}(x) \mathcal{O}_{B}(y)\right\rangle=\frac{\delta_{A B}}{(x-y)^{2 \Delta_{\mathcal{O}_{A}}}}
$$

where $\Delta_{\mathcal{O}_{A}}$ is the scaling dimension of the composite operator $\mathcal{O}_{A}$. Classically, these scaling dimensions are simply the sum of the individual dimensions of the constituent fields $\left(\left[\phi_{i}\right]=\left[A_{\mu}\right]=1\right.$ and $[\chi]=3 / 2$ ). In quantum theory the scaling dimensions receive anomalous corrections, organized in a double expansion in $\lambda=g_{\mathrm{YM}}^{2} N$ (loops) and $1 / N^{2}$ (genera)

$$
\Delta=\Delta_{0}+\sum_{l=1}^{\infty} \lambda^{l} \sum_{g=0}^{\infty} \frac{1}{N^{2 g}} \Delta_{l, g}
$$

Determining the scaling dimensions in perturbation theory is a difficult task due to the phenomenon of operator mixing: One has to identify the correct basis of (classically) degenerate gauge theory operators $\mathcal{O}_{A}$ in which (14) indeed becomes diagonal. This task is greatly facilitated through the use of the gauge theory dilatation operator, to be discussed in Section 4.

The core statement of the AdS/CFT correspondence is that the scaling dimensions $\Delta\left(\lambda, 1 / N^{2}\right)$ are equal to the energies $E$ of the $A d S_{5} \times S^{5}$ string excitations. A central problem, next to actually computing these quantities on either side of the correspondence, is to establish a "dictionary"

Living Reviews in Relativity

http: //www . livingreviews . org/lrr-2005-9 
between states in the string theory and their dual gauge theory operators. Here the underlying symmetry structure of $\mathrm{SU}(2,2 \mid 4)$ is of help, whose bosonic factors are $\mathrm{SO}(2,4) \times \mathrm{SO}(6) . \quad \mathrm{SO}(2,4)$ corresponds to the isometry group of $A d S_{5}$ or the conformal group in four dimensions respectively. $\mathrm{SO}(6)$, on the other hand, emerges from the isometries of the five sphere and the $R$-symmetry group of internal rotations of the six scalars and four gluinos in SYM. Clearly, then any state or operator can be labeled by the eigenvalues of the six Cartan generators of $\mathrm{SO}(2,4) \times \mathrm{SO}(6)$

$$
(E ; \underbrace{S_{1}, S_{2}}_{S^{3}} ; \underbrace{J_{1}, J_{2}, J_{3}}_{S^{5}})
$$

where we shall denote the $S_{i}$ as the commuting "spins" on the three sphere within $A d S_{5}$, the $J_{k}$ as the commuting angular momenta on the $S^{5}$ and $E$ is the total energy. These are the conserved quantities of the string discussed above (see Equation (8)).

The strategy is now to search for string solutions with energies

$$
E=E\left(S_{1}, S_{2}, J_{1}, J_{2}, J_{3}\right)
$$

and to identify these with scaling dimensions of Super Yang-Mills operators carrying the identical set of charges $\left(S_{1}, S_{2}, J_{1}, J_{2}, J_{3}\right)$. Generically the string energies will depend on additional data, such as winding numbers or oscillator levels, which we have suppressed in the above. 


\section{Spinning String Solutions}

We shall now look for a solution of the $A d S_{5} \times S^{5}$ string with $S_{1}=S_{2}=0$ and $J_{3}=0$, i.e. a string configuration rotating in the $S^{3}$ within $S^{5}$ and evolving only with the time coordinate of the $A d S_{5}$ space-time. This was first discussed by Frolov and Tseytlin [61, 63]. For this let us consider the following ansatz in the global coordinates of (5)

$$
t=\kappa \tau \quad \rho=0 \quad \gamma=\frac{\pi}{2} \quad \phi_{3}=0 \quad \phi_{1}=\omega_{1} \tau \quad \phi_{2}=\omega_{2} \tau \quad \psi=\psi(\sigma),
$$

with the constant parameters $\kappa, \omega_{1,2}$ and the profile $\psi(\sigma)$ to be determined. The string action (4) then becomes

$$
I=-\frac{\sqrt{\lambda}}{4 \pi} \int d \tau \int_{0}^{2 \pi} d \sigma\left[\kappa^{2}+{\psi^{\prime}}^{2}-\cos ^{2} \psi \omega_{1}^{2}-\sin ^{2} \psi \omega_{2}^{2}\right]
$$

leading to an equation of motion for $\psi(\sigma)$

$$
\psi^{\prime \prime}+\sin \psi \cos \psi\left(\omega_{2}^{2}-\omega_{1}^{2}\right)=0 .
$$

We define $\omega_{21}{ }^{2}:=\omega_{2}{ }^{2}-\omega_{1}{ }^{2}$, which we take to be positive without loss of generality. Integrating this equation once yields the "string pendulum" equation

$$
\frac{d \psi}{d \sigma}=\omega_{21} \sqrt{q-\sin ^{2} \psi},
$$

where we have introduced an integration constant $q$. Clearly, there are two qualitatively distinct situations for $q$ larger or smaller than one: For $q \leq 1$ we have a folded string with $\psi$ ranging from $-\psi_{0}$ to $\psi_{0}$, where $q=\sin ^{2} \psi_{0}$, and $\psi^{\prime}=0$ at the turning points where the string folds back onto itself (see Figure 2).

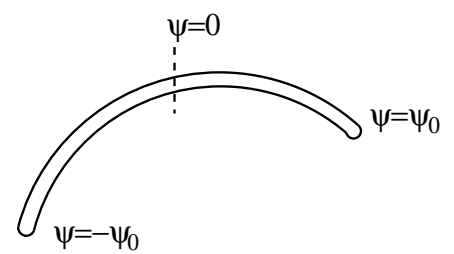

Figure 2: The folded string extending from $\psi=-\psi_{0}$ to $\psi=\psi_{0}$, where $\sin ^{2} \psi_{0}:=q$.

If, however, $q>1$ then $\psi^{\prime}$ never vanishes and we have a circular string configuration embracing a full circle on the $S^{3}$ : The energy stored in the system is large enough to let the pendulum overturn.

In addition, we have to fulfill the Virasoro constraint equations (6). One checks that our ansatz (17) satisfies the first constraint of (6), whereas the second constraint equation leads to

$$
q=\frac{\kappa^{2}-\omega_{1}^{2}}{\omega_{21}^{2}} \quad\left(\omega_{21}^{2} \neq 0\right),
$$

relating the integration constant $q$ to the parameters of our ansatz ${ }^{4}$. Our goal is to compute the energy $E$ of these two solutions as a function of the commuting angular momenta $J_{1}$ and $J_{2}$ on the three sphere within $S^{5}$. Upon using Equations (8) and inserting the ansatz (17) these are given by

$$
\begin{aligned}
E & =\sqrt{\lambda}, \\
J_{1} & =\sqrt{\lambda} \omega_{1} \int_{0}^{2 \pi} \frac{d \sigma}{2 \pi} \cos ^{2} \psi(\sigma), \quad J_{2}=\sqrt{\lambda} \omega_{2} \int_{0}^{2 \pi} \frac{d \sigma}{2 \pi} \sin ^{2} \psi(\sigma) .
\end{aligned}
$$

\footnotetext{
${ }^{4}$ The case $\omega_{21}=0$ is discussed in Section 3.1.
}

Living Reviews in Relativity

http: //www . livingreviews . org/lrr-2005-9 
From this we learn that

$$
\sqrt{\lambda}=\frac{J_{1}}{\omega_{1}}+\frac{J_{2}}{\omega_{2}}
$$

\subsection{The special case: $\omega_{1}=\omega_{2}$}

It is instructive to first discuss the particularly simple special case of a circular string, where $\omega_{1}=\omega_{2}$ and (21) does not apply. This will turn out to be a limiting case of the $q>1$ scenario. For $\omega_{21}=0$ the equation of motion for $\psi(\sigma)(19)$ immediately yields

$$
\psi^{\prime \prime}=0 \quad \Rightarrow \quad \psi(\sigma)=n \sigma
$$

with $n$ being the integer winding number of this circular string $\psi(\sigma+2 \pi)=\psi(\sigma)+2 \pi n$. In this case, the Virasoro constraints (6) yield $\kappa=\sqrt{n^{2}+\omega_{1}^{2}}$. A little bit of algebra quickly shows that the energy $E$ may be reexpressed as a function of $J_{1}=J_{2}$ and reads

$$
E=2 J_{1} \sqrt{1+\frac{n^{2} \lambda}{4 J_{1}^{2}}}
$$

which is analytic in $\frac{\lambda}{J_{1}^{2}}$ ! This amounts to an all-loop prediction for the dual gauge theory scaling dimension in the BMN limit $J_{1} \rightarrow \infty$ with $\lambda / J_{1}{ }^{2}$ fixed, quite similar to the result for the plane-wave superstring discussed above.

Let us now discuss the folded and circular string solutions in turn.

\subsection{The folded string: $q \leq 1$}

In the folded case $J_{1}$ may be expressed in terms of an elliptic integral ${ }^{5}$ by substituting (using (20))

$$
d \sigma=\frac{d \psi}{\omega_{21} \sqrt{q-\sin ^{2} \psi}}
$$

into (23) and performing some elementary transformations to find $\left(q=\sin ^{2} \psi_{0}\right)$

$$
J_{1}=\frac{\sqrt{\lambda} \omega_{1}}{2 \pi} 4 \int_{0}^{\psi_{0}} d \psi \frac{\cos ^{2} \psi}{\omega_{21} \sqrt{\sin ^{2} \psi_{0}-\sin ^{2} \psi}}=\frac{2 \sqrt{\lambda} \omega_{1}}{\pi \omega_{21}} E\left(\sin ^{2} \psi_{0}\right),
$$

where we only need to integrate over one quarter of the folded string due to symmetry considerations (see Figure 2). Additionally, we have

$$
2 \pi=\int_{0}^{2 \pi} d \sigma=4 \int_{0}^{\psi_{0}} \frac{d \psi}{\omega_{21} \sqrt{\sin ^{2} \psi_{0}-\sin ^{2} \psi}}=\frac{4}{\omega_{21}} K\left(\sin ^{2} \psi_{0}\right) .
$$

The four Equations $(21,24,28,29)$ may then be used to eliminate the parameters of our solution $\kappa, \omega_{1}$ and $\omega_{2}$. For this, rewrite (28) and (29) as $(\kappa=E / \sqrt{\lambda})$

$$
\frac{\omega_{1}}{\omega_{21}}=\frac{\pi}{2 \sqrt{\lambda}} \frac{J_{1}}{E(q)}, \quad \frac{\kappa}{\omega_{21}}=\frac{\pi}{2 \sqrt{\lambda}} \frac{E}{K(q)},
$$

\footnotetext{
${ }^{5}$ Our conventions are $(x<1)$

$$
E(x):=\int_{0}^{\pi / 2} d \psi \sqrt{1-x \sin ^{2} \psi} \quad K(x):=\int_{0}^{\pi / 2} d \psi \frac{1}{\sqrt{1-x \sin ^{2} \psi}} .
$$
}


and use (24) to deduce

$$
\frac{\omega_{2}}{\omega_{21}}=\frac{\pi}{2 \sqrt{\lambda}} \frac{J_{2}}{K(q)-E(q)} .
$$

Then, the Virasoro constraint equation (21) and the identity $1=\left(\omega_{2}^{2}-\omega_{1}^{2}\right) / \omega_{21}{ }^{2}$ yield the two folded string equations

$$
\frac{4 q \lambda}{\pi^{2}}=\frac{E^{2}}{K(q)^{2}}-\frac{J_{1}^{2}}{E(q)^{2}}, \quad \frac{4 \lambda}{\pi^{2}}=\frac{J_{2}{ }^{2}}{(K(q)-E(q))^{2}}-\frac{J_{1}{ }^{2}}{E(q)^{2}},
$$

which implicitly define the sought after energy function $E=E\left(J_{1}, J_{2}\right)$ upon further elimination of $q$. This is achieved by assuming an analytic behavior of $q$ and $E$ in the BMN type limit of large total angular momentum $J:=J_{1}+J_{2} \rightarrow \infty$, with $\lambda / J^{2}$ held fixed

$$
\begin{aligned}
q & =q_{0}+\frac{\lambda}{J^{2}} q_{1}+\frac{\lambda^{2}}{J^{4}} q_{2}+\cdots \\
E & =J\left(E_{0}+\frac{\lambda}{J^{2}} E_{1}+\frac{\lambda^{2}}{J^{4}} E_{2}+\cdots\right) .
\end{aligned}
$$

Plugging these expansions into (32) one can solve for the $q_{i}$ and $E_{i}$ iteratively. At leading order $E_{0}=1$ (as it should from the dual gauge theory perspective) and $q_{0}$ is implicitly determined through the "filling fraction" $J_{2} / J$

$$
\frac{J_{2}}{J}=1-\frac{E\left(q_{0}\right)}{K\left(q_{0}\right)} .
$$

The first non-trivial term in the energy is then expressed in terms of $q_{0}$ through

$$
E_{1}=\frac{2}{\pi^{2}} K\left(q_{0}\right)\left(E\left(q_{0}\right)-\left(1-q_{0}\right) K\left(q_{0}\right)\right)
$$

yielding a clear prediction for one-loop gauge theory. The higher order $E_{i}$ can then also be obtained. To give a concrete example, let us evaluate $E$ for the first few orders in $\lambda / J^{2}$ in the "half filled" case $J_{1}=J_{2}$ :

$$
E=2 J_{1}\left(1+\frac{0.71}{8} \frac{\lambda}{J_{1}{ }^{2}}-\frac{1.69}{32} \frac{\lambda^{2}}{J_{1}{ }^{4}}+\cdots\right)
$$

which shows that the energy of the folded string configuration is smaller than the one of the closed configuration of (26) for single winding $n=1$.

\subsection{The circular string: $q>1$}

For $q>1$ the string does not fold back onto itself and extends around the full circle of $\psi \in[0,2 \pi]$ as $\sigma$ runs from 0 to $2 \pi$. The initial expression for (28) now changes only marginally to

$$
J_{1}=\frac{\sqrt{\lambda} \omega_{1}}{2 \pi} \int_{0}^{2 \pi} d \psi \frac{\cos ^{2} \psi}{\omega_{21} \sqrt{q-\sin ^{2} \psi}} .
$$

Elementary transformations yield

$$
J_{1}=\frac{2 \sqrt{\lambda} \omega_{1}}{\pi \omega_{21}}\left[\frac{1-q}{\sqrt{q}} K\left(q^{-1}\right)+\sqrt{q} E\left(q^{-1}\right)\right] .
$$

Analogously (29) now becomes

$$
2 \pi=\int_{0}^{2 \pi} d \sigma=4 \int_{0}^{\pi / 2} \frac{d \psi}{\omega_{21} \sqrt{\sin ^{2} \psi_{0}-\sin ^{2} \psi}}=\frac{4}{\omega_{21} \sqrt{q}} K\left(q^{-1}\right) .
$$

Living Reviews in Relativity

http: //www . livingreviews . org/lrr-2005-9 
The corresponding relations to (30) and (31) then take the form

$$
\begin{aligned}
\frac{1}{\omega_{21}} & =\frac{\pi}{2} \frac{\sqrt{q}}{K\left(q^{-1}\right)}, \quad \frac{\omega_{1}}{\omega_{21}}=\frac{\pi}{2 \sqrt{\lambda}} \frac{\sqrt{q} J_{1}}{(1-q) K\left(q^{-1}\right)+q E(q)}, \\
\frac{\omega_{2}}{\omega_{21}} & =\frac{\pi}{2 \sqrt{\lambda}} \frac{J_{2}}{\sqrt{q}\left[K\left(q^{-1}\right)-E\left(q^{-1}\right)\right]} .
\end{aligned}
$$

From these, one deduces in complete analogy to (32) the two circular string equations

$$
\begin{gathered}
\frac{4 \lambda}{\pi^{2}}=\frac{E^{2}}{K\left(q^{-1}\right)^{2}}-\frac{J_{1}{ }^{2}}{\left[(1-q) K\left(q^{-1}\right)+q E\left(q^{-1}\right)\right]^{2}} \\
\frac{4 q \lambda}{\pi^{2}}=\frac{J_{2}{ }^{2}}{\left[K\left(q^{-1}\right)-E\left(q^{-1}\right)\right]^{2}}-\frac{q^{2} J_{1}{ }^{2}}{\left[(1-q) K\left(q^{-1}\right)+q E\left(q^{-1}\right)\right]^{2}},
\end{gathered}
$$

which encode the energy relation $E=E\left(J_{1}, J_{2}\right)$ upon elimination of $q$. In order to do this, we again make an analytic ansatz in $\lambda / J^{2}$ for $q$ and $E$ as we did in (33). This yields the following implicit expression for $q_{0}$ in terms of the filling fraction $J_{2} / J$

$$
\frac{J_{2}}{J}=q_{0}\left(1-\frac{E\left(q_{0}^{-1}\right)}{K\left(q_{0}^{-1}\right)}\right)
$$

The first two energy terms in the $\lambda / J^{2}$ expansion then take the form

$$
E_{0}=1, \quad E_{1}=\frac{2}{\pi^{2}} E\left(q_{0}^{-1}\right) K\left(q_{0}^{-1}\right),
$$

which again gives a clean prediction for the dual gauge theory scaling dimensions at one-loop.

In Figure 3, we have plotted the energies of the folded and circular string solutions against the filling fraction $J_{2} / J$. As expected from the "string pendulum" picture of (20), the folded string solution has lower energy for fixed filling fraction $J_{2} / J$ : The pendulum does not perform a full turn, but oscillates back and forth. Note that the folded string approaches $E_{1}=0$ in the limit $J_{2} / J \rightarrow 0$, which is the rotating point-particle solution of Section 2.1. The quantum fluctuations about it correspond to the plane-wave string domain. Note also that the simplest circular string solution with $\omega_{1}=\omega_{2}$ discussed in Section 3.1 is the minimum $\left(J_{2} / J=0.5\right)$ of the full circular string solution for $n=1$.

These classical string energies will be reproduced in a dual one-loop gauge theory computation in Section 4.

\subsection{Further Developments}

The discussed explicit solutions of the bosonic string on a $\mathbb{R} \times S^{3}$ background are the first simple elements of the general set of classical solutions of the bosonic $A d S_{5} \times S^{5}$ string considered in the literature. Earlier examples not discussed in the text are [69, 102, 91]. The bosonic part of the classical string action consists of a $\mathrm{SO}(6)$ and a $\mathrm{SO}(2,4) \sigma$-model augmented by the conformal gauge (Virasoro) constraints. The $O(p, q) \sigma$-models have been known to be integrable for a long time $[98,85,118,57]$ and one would expect this to remain true also once one imposes the conformal gauge constraints. And, indeed, in [5, 7] more complicated solutions of the $A d S_{5} \times S^{5}$ string were constructed by reducing the system to the so-called Neumann integrable system through a suitable ansatz. The solutions of $[5,7]$ involve non-vanishing values for all spins and angular momenta $\left(S_{1}, S_{2}, J_{1}, J_{2}, J_{3}\right)$. However, they do not generically display an analytic behavior in the effective coupling constant $\lambda^{\prime}$. This is true only for configurations with at least one large charge $J_{i}$ on the $S^{5}$. 


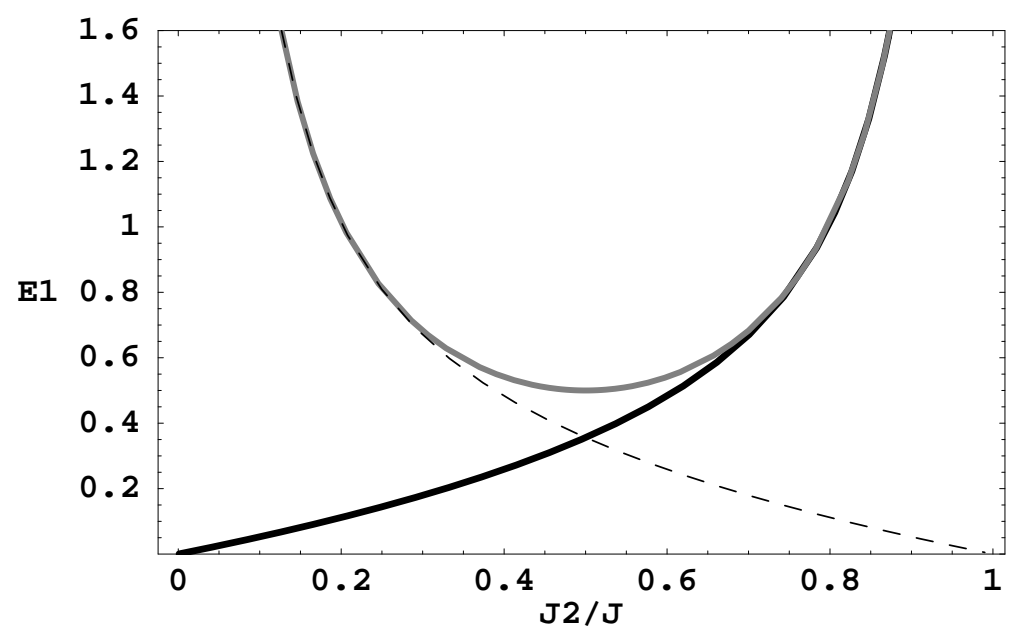

Figure 3: The one-loop energies of the folded (dark) and circular (light) string solutions plotted against the filling fraction $J_{2} / J$. The dashed curve is the mirrored folded string solution where one interchanges $J_{1} \leftrightarrow J_{2}$.

In the context of the $O(p, q) \sigma$-models, integrability is based on the existence of a Lax pair, a family of flat connections on the $2 \mathrm{~d}$ string worldsheet, giving rise to an infinite number of conserved charges. These were first discussed in the context of the bosonic string in [87] and for the full superstring in [31]. The Lax pair for the string was put to use in [74] for string configurations on $\mathbb{R} \times S^{3}$ - the sector we also considered in the above. These investigations led to the construction of an underlying algebraic curve parameterizing the solutions. This enabled the authors of [74] to write down an integral equation of Bethe type yielding the associated energies of the solutions. Very similar equations will appear below in our discussion in Section 4.2 on the thermodynamic limit of the gauge theory Bethe equations. The extraction of these integral equations from the string $\sigma$-model then allows for a direct comparison to the gauge theory Bethe equations. On this level of formalization, there is no need to compare explicit solutions any longer - as we are doing here for pedagogical purposes. This construction based on an underlying algebraic curve makes full use of the technology of integrable systems and has been nicely reviewed by Zarembo in [119].

In the very interesting paper [6], these continuum string Bethe equations were boldly discretized leading to a conjectured set of Bethe equations for the quantum spectrum of the string. This proposal has been verified by comparing it to known quantum data of the $A d S_{5} \times S^{5}$ string: The near plane-wave spectrum of the superstring of [46, 45, 44, 43], as well as the expected [68] generic scaling of the string energies with $\lambda^{1 / 4}$ in the strong coupling limit, agree with the predictions of the quantum string Bethe equations. But there is more quantum data for the $A d S_{5} \times S^{5}$ string available: In a series of papers by Tseytlin, Frolov and collaborators, one-loop corrections on the string worldsheet to the energies of various spinning string solutions have been computed [62, 64, 95]. The one-loop correction for a circular string moving in $A d S_{3} \times S^{1} \subset A d S_{5} \times S^{5}$ obtained in [95] was recently compared [106] to the result obtained from the proposed quantum string Bethe equations of [6]. The authors of [106] find agreement when they expand the results in $\lambda^{\prime}$ (up to third order), but disagreements emerge in different limits (where $\lambda^{\prime}$ is not small). The interpretation of this result is unclear at present. Finally, the proposed quantum string Bethe equations of [6] can also be microscopically attributed to a $s=1 / 2$ spin chain model with long-range interactions up to (at least) order five in a small $\lambda$ expansion [14].

The technically involved construction of algebraic curves solving the classical $\mathbb{R} \times S^{3}$ string

Living Reviews in Relativity

http://www. livingreviews.org/lrr-2005-9 
$\sigma$-model has subsequently been generalized to larger sectors: In [17] to $\mathbb{R} \times S^{5}$ (or $\mathrm{SO}(6)$ in gauge theory language) configurations, in [75] to $A d S_{3} \times S^{1}$ (or SL(2)) string configurations, and finally in [18] to superstrings propagating in the full $A d S_{5} \times S^{5}$ space.

There also has been progress on a number of possible paths towards the true quantization of the classical integrable model of the $A d S_{5} \times S^{5}$ string in the works [113, 4, 2, 9, 35, 36], however, it is fair to say that this problem remains currently unsolved. 


\section{The Dual Gauge Theory Side}

Let us now turn to the identification of the folded and circular string solutions in the dual gauge theory.

Our aim is to reproduce the obtained energy functions $E_{1}\left(J_{1}, J_{2}\right)$ plotted in Figure 3 from a dual gauge theory computation at one-loop. For this, we need to identify the gauge theory operators, which are dual to the spinning strings on $\mathbb{R} \times S^{3}$. As here $J_{2}=0=S_{1}=S_{2}$ the relevant operators will be built from the two complex scalars $Z:=\phi_{1}+i \phi_{2}$ and $W:=\phi_{3}+i \phi_{4}$ with a total number of $J_{1} Z$-fields and $J_{2} W$-fields, i.e.

$$
\mathcal{O}_{\alpha}^{J_{1}, J_{2}}=\operatorname{Tr}\left[Z^{J_{1}} W^{J_{2}}\right]+\cdots
$$

where the dots denote suitable permutations of the $Z$ and $W$ to be discussed. An operator of the form (44) may be pictured as a ring of black (" $Z$ ") and red (" $W$ ") beads - or equivalently as a configuration of an $s=1 / 2$ quantum spin chain, where $W$ corresponds to the state $|\uparrow\rangle$ and $Z$ to $|\downarrow\rangle$.

$$
\operatorname{Tr}\left[Z W^{2} Z W^{4}\right] \Leftrightarrow
$$

How does one compute the associated scaling dimensions at (say) one loop order for $J_{1}, J_{2} \rightarrow \infty$ ? Clearly one is facing a huge operator mixing problem as all $\mathcal{O}_{i}^{J_{1}, J_{2}}$ with arbitrary permutations of $Z$ 's and $W$ 's are degenerate at tree level where $\Delta_{0}^{\mathcal{O}_{i}^{J_{1}, J_{2}}}=J_{1}+J_{2}$.

A very efficient tool to deal with this problem is the dilatation operator $\mathcal{D}$, which was introduced in $[20,21]$. It acts on the trace operators $\mathcal{O}_{\alpha}^{J_{1}, J_{2}}$ at a fixed space-time point $x$ and its eigenvalues are the scaling dimensions $\Delta$

$$
\mathcal{D} \circ \mathcal{O}_{\alpha}^{J_{1}, J_{2}}(x)=\sum_{\beta} \mathcal{D}_{\alpha \beta} \mathcal{O}_{\beta}^{J_{1}, J_{2}}(x)
$$

The dilatation operator is constructed in such a fashion as to attach the relevant diagrams to the open legs of the

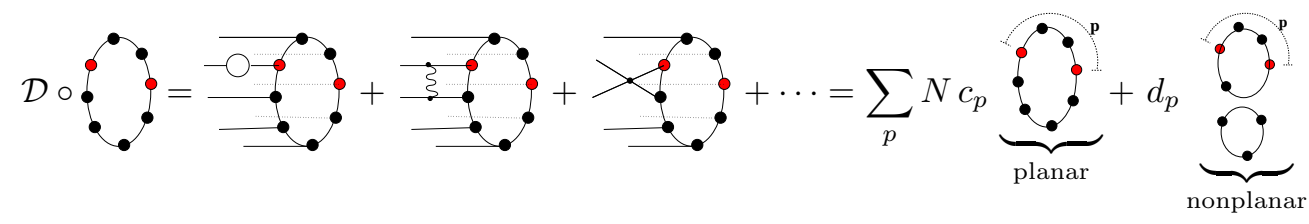

Figure 4: The action of the dilatation operator on a trace operator. theory

"incoming" trace operators (as depicted in Figure 4) and may be computed in perturbation

$$
\mathcal{D}=\sum_{n=0}^{\infty} \mathcal{D}^{(n)}
$$

where $\mathcal{D}^{(n)}$ is of order $g_{\mathrm{YM}}^{2 n}$. For the explicit computation of the one-loop piece $\mathcal{D}^{(1)}$ see e.g. the review [97], where the concrete relation to two-point functions is also explained. In our "minimal" $\mathrm{SU}(2)$ sector of complex scalar fields $Z$ and $W$ it takes the rather simple form

$$
\mathcal{D}^{(0)}=\operatorname{Tr}(Z \check{Z}+W \check{W}), \quad \mathcal{D}^{(1)}=-\frac{g_{\mathrm{YM}}^{2}}{8 \pi^{2}} \operatorname{Tr}[Z, W][\check{Z}, \check{W}], \quad \text { where } \quad \check{Z}_{i j}:=\frac{d}{d Z_{j i}} .
$$

Living Reviews in Relativity

http: //www. livingreviews.org/lrr-2005-9 
Note that the tree-level piece $\mathcal{D}^{(0)}$ simply measures the length of the incident operator (or spin chain) $J_{1}+J_{2}$. The eigenvalues of the dilatation operator then yield the scaling dimensions we are looking for - diagonalization of $\mathcal{D}$ solves the mixing problem.

We shall be exclusively interested in the planar contribution to $\mathcal{D}$, as this sector of the gauge theory corresponds to the "free" (in the sense of $g_{s}=0$ ) $A d S_{5} \times S^{5}$ string. For this, it is important to realize that the planar piece of $\mathcal{D}^{(1)}$ only acts on two neighboring fields in the chain of $Z$ 's and $W^{\prime}$ 's. This may be seen by evaluating explicitly the action of $\mathcal{D}^{(1)}$ on two fields $Z$ and $W$ separated by arbitrary matrices $\mathcal{A}$ and $\mathcal{B}$

$$
\operatorname{Tr}[Z, W][\check{Z}, \check{W}] \circ \operatorname{Tr}(Z \mathcal{A} W \mathcal{B})=-\operatorname{Tr}(\mathcal{A}) \operatorname{Tr}([Z, W] \mathcal{B})+\operatorname{Tr}(\mathcal{B}) \operatorname{Tr}([Z, W] \mathcal{A}) .
$$

Clearly, there is an enhanced contribution when $\mathcal{A}=1$ or $\mathcal{B}=1$, i.e. $Z$ and $W$ are nearest neighbors on the spin chain. From the above computation we learn that

$$
\mathcal{D}_{\text {planar }}^{(1)}=\frac{\lambda}{8 \pi^{2}} \sum_{i=1}^{L}\left(\mathbf{1}_{i, i+1}-P_{i, i+1}\right)
$$

where $P_{i, j}$ permutes the fields (or spins) at sites $i$ and $j$ and periodicity $P_{L, L+1}=P_{1, L}$ is understood. Remarkably, as noticed by Minahan and Zarembo [92], this spin chain operator is the Heisenberg $\mathrm{XXX}_{1 / 2}$ quantum spin chain Hamiltonian, which is the prototype of an integrable spin chain. Written in terms of the Pauli matrices $\vec{\sigma}_{i}$ acting on the spin at site $i$ one finds

$$
\mathcal{D}_{\text {planar }}^{(1)}=\frac{\lambda}{8 \pi^{2}} \mathcal{H}_{\mathrm{Xxx}_{1 / 2}}=\frac{\lambda}{4 \pi^{2}} \sum_{i=1}^{L}\left(\frac{1}{4}-\vec{\sigma}_{i} \cdot \vec{\sigma}_{i+1}\right) .
$$

Due to the positive sign of the sum, the spin chain is ferromagnetic and its ground state is $\mid \downarrow \downarrow$ $\ldots \downarrow\rangle_{\text {cyclic }} \Leftrightarrow \operatorname{Tr}\left(Z^{L}\right)$ : the gauge dual of the rotating point particle of Section 2.1. Excitations of the ground state are given by spin flips or "magnons". Note that a one-magnon excitation $|\downarrow \ldots \downarrow \uparrow \downarrow \ldots \downarrow\rangle_{\text {cyclic }}$ has vanishing energy due to the cyclic property of the trace, it corresponds to a zero mode plane-wave string excitation $\alpha_{0}^{\dagger}|0\rangle$. Two-magnon excitations are the first stringy excitations which are dual to the $\alpha_{n}^{\dagger} \alpha_{-n}^{\dagger}|0\rangle$ state of the plane-wave string in the BMN limit.

The integrability of the spin chain amounts to the existence of $L-1$ higher charges $Q_{k}$ which commute with the Hamiltonian (alias dilatation operator) and amongst themselves, i.e. $\left[Q_{k}, Q_{l}\right]=$ 0 . Explicitly the first two charges of the Heisenberg chain are given by

$$
Q_{2}:=\mathcal{H}_{\mathrm{Xxx}_{1 / 2}} \quad Q_{3}=\sum_{i=1}^{L}\left(\vec{\sigma}_{i} \times \vec{\sigma}_{i+1}\right) \cdot \vec{\sigma}_{i+2} .
$$

The explicit form of all the higher $Q_{k}$ may be found in [67]. Note that $Q_{k}$ will involve up to $k$ neighboring spin interactions.

\subsection{The coordinate Bethe ansatz}

We now discuss the ansatz that enabled Bethe to diagonalize the Heisenberg model in $1931[37]^{6}$. For this, we will drop the cyclicity constraint imposed on us from the underlying trace structure of the gauge theory operators for the moment, and treat a general non-cyclic, but periodic, spin chain. The vacuum state of the Heisenberg chain is then given by $|\downarrow \ldots \downarrow\rangle$. Let $\left|x_{1}, x_{2}, \ldots, x_{J}\right\rangle$ with $x_{1}<x_{2}<\cdots<x_{J}$ denote a state of the chain with up-spins (magnons) located at sites $x_{i}$,

\footnotetext{
${ }^{6}$ For a nice and detailed review on this topic see [73]. The technology of the algebraic Bethe ansatz is reviewed in [55].
} 
i.e. $|1,3,4\rangle_{L=5}=|\downarrow \uparrow \downarrow \downarrow \uparrow\rangle$. It is useful to think of these spin flips as particles located at the sites $x_{i}$. Note that the Hamiltonian preserves the magnon or particle number.

The one magnon sector is then trivially diagonalized by Fourier transformation

$$
\begin{aligned}
\left|\psi\left(p_{1}\right)\right\rangle:= & \sum_{x=1}^{L} e^{i p_{1} x}|x\rangle, \quad \text { with } \quad Q_{2}\left|\psi\left(p_{1}\right)\right\rangle=4 \sin ^{2}\left(\frac{p_{1}}{2}\right)\left|\psi\left(p_{1}\right)\right\rangle \\
& \text { where } \quad Q_{2}=\sum_{i=1}^{L}\left(\mathbf{1}_{i, i+1}-P_{i, i+1}\right)
\end{aligned}
$$

as $2-e^{i p}-e^{-i p}=4 \sin ^{2}(p / 2)$. The periodic boundary conditions require the one-magnon momenta to be quantized $p_{1}=2 \pi k / L$ with $k \in \mathbb{Z}$.

Next, consider a general two-magnon state of the form

$$
\left|\psi\left(p_{1}, p_{2}\right)\right\rangle=\sum_{1 \leq x_{1}<x_{2} \leq L} \psi\left(x_{1}, x_{2}\right)\left|x_{1}, x_{2}\right\rangle .
$$

with a two-particle wave-function $\psi\left(x_{1}, x_{2}\right)$. The "position space" Schrödinger equation following from $\sum_{i=1}^{L}\left(1-P_{i, i+1}\right)\left|\psi\left(p_{1}, p_{2}\right)\right\rangle=E_{2}\left|\psi\left(p_{1}, p_{2}\right)\right\rangle$ then leads to two sets of equations, depending on whether the particles lie next to each other or not:

$$
\begin{aligned}
x_{2}>x_{1}+1 \quad E_{2} \psi\left(x_{1}, x_{2}\right) & =2 \psi\left(x_{1}, x_{2}\right)-\psi\left(x_{1}-1, x_{2}\right)-\psi\left(x_{1}+1, x_{2}\right) \\
& +2 \psi\left(x_{1}, x_{2}\right)-\psi\left(x_{1}, x_{2}-1\right)-\psi\left(x_{1}, x_{2}+1\right) \\
x_{2}=x_{1}+1 \quad E_{2} \psi\left(x_{1}, x_{2}\right) & =2 \psi\left(x_{1}, x_{2}\right)-\psi\left(x_{1}-1, x_{2}\right)-\psi\left(x_{1}, x_{2}-1\right) .
\end{aligned}
$$

$E_{2}$ is the eigenvalue of $Q_{2}$ and related to the gauge theory scaling dimensions as $\Delta=L+\frac{\lambda}{8 \pi^{2}} E_{2}+$ $\mathcal{O}\left(\lambda^{2}\right)$. The above equations can be fulfilled by a superposition ansatz with an incoming and outgoing plane wave (Bethe's ansatz)

$$
\psi\left(x_{1}, x_{2}\right)=e^{i\left(p_{1} x_{1}+p_{2} x_{2}\right)}+S\left(p_{2}, p_{1}\right) e^{i\left(p_{2} x_{1}+p_{1} x_{2}\right)},
$$

where $S\left(p_{1}, p_{2}\right)$ denotes the S-matrix of the scattered particles. Note that in the second term describing the scattered contribution, the two particles have simply exchanged their momenta. One easily sees that (55) is fulfilled for an arbitrary $S\left(p_{2}, p_{1}\right)$ yielding the energy as a sum of one-particle energies

$$
E_{2}=4 \sin ^{2}\left(\frac{p_{1}}{2}\right)+4 \sin ^{2}\left(\frac{p_{2}}{2}\right) .
$$

Equation (56) then determines the S-matrix to be of the form

$$
S\left(p_{1}, p_{2}\right)=\frac{\varphi\left(p_{1}\right)-\varphi\left(p_{2}\right)+i}{\varphi\left(p_{1}\right)-\varphi\left(p_{2}\right)-i} \quad \text { with } \quad \varphi(p)=\frac{1}{2} \cot \left(\frac{p}{2}\right) .
$$

Note that $S\left(p_{1}, p_{2}\right)^{-1}=S\left(p_{2}, p_{2}\right)$. This solves the infinite length chain. For a finite chain, the momenta $p_{i}$ are no longer arbitrary continuous quantities, but become discrete through the periodic boundary condition

$$
\psi\left(x_{1}, x_{2}\right)=\psi\left(x_{2}, x_{1}+L\right) .
$$

This, in turn, leads to the Bethe equations for the two magnon problem

$$
e^{i p_{1} L}=S\left(p_{1}, p_{2}\right) \quad \text { and } \quad e^{i p_{2} L}=S\left(p_{2}, p_{1}\right)
$$

implying $p_{1}+p_{2}=2 \pi m$ with an arbitrary integer $m$. The solutions of the algebraic equations (61) for $p_{1}$ and $p_{2}$ then determine the corresponding energies by plugging the resulting quasi-momenta $p_{i}$ into (58).

Living Reviews in Relativity

http: //www . livingreviews . org/lrr-2005-9 
The magic of integrability now is that this information is all that is needed to solve the general $M$-body problem! This phenomenon is known as factorized scattering: The multi-body scattering process factorizes into a sequence of two-body interactions under which two incoming particles of momenta $p_{i}$ and $p_{j}$ scatter off each other elastically with the S-matrix $S\left(p_{j}, p_{i}\right)$, thereby simply exchanging their momenta. That is, the $M$-body wave-function takes the form [37, 73]

$$
\psi\left(x_{1}, \ldots, x_{M}\right)=\sum_{P \in \operatorname{Perm}(M)} \exp \left[i \sum_{i=1}^{M} p_{P(i)} x_{i}+\frac{i}{2} \sum_{i<j} \theta_{P(i) P(j)}\right]
$$

where the sum is over all $M$ ! permutations of the labels $\{1,2, \ldots, M\}$ and the phase shifts $\theta_{i j}=-\theta_{j i}$ are related to the S-matrix (59) by

$$
S\left(p_{i}, p_{j}\right)=\exp \left[i \theta_{i j}\right] .
$$

The $M$-magnon Bethe ansatz then yields the set of $M$ Bethe equations

$$
e^{i p_{k} L}=\prod_{i=1, i \neq k}^{M} S\left(p_{k}, p_{i}\right)
$$

with the two-body S-matrix of (59) and the additive energy expression

$$
E_{2}=\sum_{i=1}^{M} 4 \sin ^{2}\left(\frac{p_{i}}{2}\right)
$$

In order to reinstate the cyclicity of the trace condition, one needs to further impose the constraint of a total vanishing momentum

$$
\sum_{i=1}^{M} p_{i}=0
$$

As an example, let us diagonalize the two magnon problem exactly. Due to (66), we have $p:=p_{1}=-p_{2}$ and the Bethe equations (61) reduce to the single equation

$$
e^{i p L}=\frac{\cot \frac{p}{2}+i}{\cot \frac{p}{2}-i}=e^{i p} \quad \Rightarrow \quad e^{i p(L-1)}=1 \quad \Rightarrow \quad p=\frac{2 \pi n}{L-1} .
$$

The energy eigenvalue then reads

$$
E_{2}=8 \sin ^{2}\left(\frac{\pi n}{L-1}\right) \stackrel{L \rightarrow \infty}{\longrightarrow} 8 \pi^{2} \frac{n^{2}}{L^{2}},
$$

which upon reinserting the dropped prefactor of $\frac{\lambda}{8 \pi^{2}}$ yields the one-loop scaling dimension $\Delta^{(1)}=$ $\frac{\lambda}{\pi^{2}} \sin ^{2}\left(\frac{\pi n^{2}}{L^{2}}\right)$ of the two-magnon operators [10, 92]

$$
\mathcal{O}_{n}^{(J, 2)}=\sum_{p=0}^{J} \cos \left(\pi n \frac{2 p+1}{J+1}\right) \operatorname{Tr}\left(W Z^{p} W Z^{J-p}\right) .
$$

In the BMN limit $N, J \rightarrow \infty$ with $\lambda / J^{2}$ fixed, the scaling dimension takes the famous value $\Delta^{(1)}=n^{2} \lambda / J^{2}$, corresponding to the first term in the expansion of the level-two energy spectrum of the plane-wave superstring $E_{\text {light-cone }}=\sqrt{1+n^{2} \lambda / J^{2}}[33]$.

Hence, from the viewpoint of the spin chain, the plane-wave limit corresponds to a chain of diverging length $L \gg 1$ carrying a finite number of magnons $M$, which are nothing but the gauge duals of the oscillator excitations of the plane-wave superstring. 


\subsection{The thermodynamic limit of the spin chain}

In order to make contact with the spinning string solution discussed in Section 3, we will now consider the thermodynamic limit of the spin chain, in which the length $L$ and the number of magnons $M$ become large. This is necessary, as the classical string solutions only limit to the true quantum result in the BMN type limit $J_{2}, J \rightarrow \infty$ with the filling fraction $J_{2} / J$ held fixed (here $J_{2}=M$ and $J=L$ ). This thermodynamic groundstate solution of the gauge theory Bethe equations was found in $[22,16,8,54]$, which we closely follow.

For this, it is useful to reexpress the Bethe equations (64) in terms of the Bethe roots $u_{k}$ related to the momenta via

$$
u_{k}=\frac{1}{2} \cot \frac{p_{k}}{2}
$$

for which the Bethe equations (64) and the momentum constraint (66) become

$$
\left(\frac{u_{i}+i / 2}{u_{i}-i / 2}\right)^{L}=\prod_{k \neq i}^{M} \frac{u_{i}-u_{k}+i}{u_{i}-u_{k}-i}, \quad \prod_{i=1}^{M} \frac{u_{i}+i / 2}{u_{i}-i / 2}=1 .
$$

The energy then is

$$
Q_{2}=\sum_{i=1}^{M} \frac{1}{u_{i}^{2}+\frac{1}{4}} .
$$

The momentum constraint can be satisfied by considering symmetric root distributions of the form $\left(u_{i},-u_{i}, u_{i}^{*},-u_{i}^{*}\right)$. The thermodynamic limit is now obtained by first taking the logarithm of (71)

$$
L \ln \left(\frac{u_{i}+i / 2}{u_{i}-i / 2}\right)=\sum_{k=1}^{M} \ln \left(\frac{u_{i}-u_{k}+i}{u_{i}-u_{k}-i}\right)-2 \pi i n_{i}
$$

where $n_{i}$ is an arbitrary integer associated to every root $u_{i}$. One self-consistently assumes that the momenta scale as $p_{i} \sim 1 / L$ for $L \rightarrow \infty$ implying that the Bethe roots scale as $u_{i} \sim L$. Therefore, in the $L \rightarrow \infty$ limit the above equation reduces to

$$
\frac{1}{u_{i}}=2 \pi n_{j}+\frac{2}{L} \sum_{k=1(k \neq i)}^{M} \frac{1}{u_{j}-u_{k}} .
$$

In the thermodynamic limit, the roots $u_{i}$ accumulate on smooth contours in the complex plane known as "Bethe strings," which turn the set of algebraic Bethe equations into an integral equation. To see this, introduce the Bethe root density

$$
\rho(u):=\frac{1}{M} \sum_{j=1}^{M} \delta\left(u-u_{j}\right) \quad \text { with } \quad \int_{C} d u \rho(u)=1
$$

where $C$ is the support of the density, i.e. the union of all Bethe string contours. Multiplying (74) with $u_{i}$ and introducing $\rho(u)$, one arrives at the singular integral equation ${ }^{7}$

$$
f_{C} d v \frac{\rho(v) u}{v-u}=-\frac{1}{2 \alpha}+\frac{\pi n_{C(u)} u}{\alpha} \quad \text { where } \quad u \in C \quad \text { and } \quad \alpha:=\frac{M}{L} .
$$

The mode numbers $n_{C(u)}$ are integers, which are assumed to be constant on each smooth component $C_{n}$ of the density support $C=\cup C_{n}$ in the complex plane. These integers and the distribution of

${ }^{7} f d v \frac{(\ldots)}{v-u}$ denotes the principle part prescription.

Living Reviews in Relativity

http: //www . livingreviews . org/lrr-2005-9 
components $C_{n}$ select the numerous solutions to the continuum Bethe equations (76). Furthermore, the continuum energy now becomes

$$
Q_{2}=M \int_{C} \frac{\rho(u)}{u^{2}} .
$$

As was shown in [22], the gauge dual to the folded string solution of Section 3.2 corresponds to a two-cut support $C=C_{1} \cup C_{1}^{*}$, with $n_{C_{1}}=-1$ and $n_{C_{1}^{*}}=1$, sketched in Figure 5 .

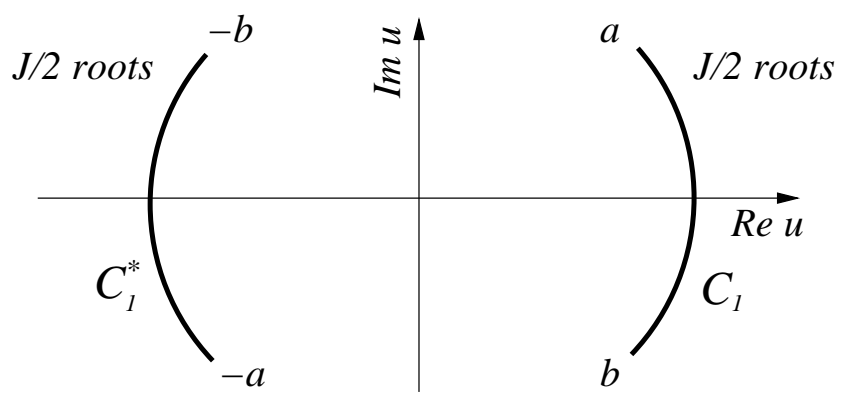

Figure 5: Bethe root distribution for the gauge dual of the folded string. For large $L$ the roots condense into two cuts in the complex plane.

The key trick to obtain analytical expressions for $\rho(u)$ is to consider the analytic continuation to negative filling fraction $\beta:=-\alpha$ : Then the two cuts $C_{1} \cup C_{1}^{*}$ are mapped to intervals on the real line $\left(C_{1}^{*} \rightarrow[-b,-a]\right.$ and $\left.C_{1} \rightarrow[a, b]\right)[22]$. Then (76) may be brought into the compact form

$$
f_{a}^{b} d v \frac{\tilde{\rho}(v) u^{2}}{v^{2}-u^{2}}=\frac{1}{4}-\frac{\pi u}{2} \quad \text { with } \quad \int_{a}^{b} d v \tilde{\rho}(v)=\frac{\beta}{2},
$$

using $\rho(-v)=\rho(v)$ and defining $\tilde{\rho}(v):=\beta \rho(v)$. In order to proceed, one introduces the resolvent

$$
H(u):=\int_{a}^{b} d v \tilde{\rho}(v) \frac{v^{2}}{v^{2}-u^{2}}=-\frac{\alpha}{2}+\sum_{k=1}^{\infty} Q_{2 k} u^{2 k}
$$

which gives rise to the infinite tower of conserved even charges $Q_{2 k}$ with the energy $E_{2}=\frac{1}{8 \pi^{2}} Q_{2}$ [8]. Across the cut $u \in[a, b]$ the resolvent $H(u)$ behaves as

$$
H(u \pm i \epsilon)=-\frac{\alpha}{2}+\frac{1}{4}-\frac{\pi}{2} u \pm i \pi \frac{u}{2} \tilde{\rho}(u), \quad u \in[a, b],
$$

which one shows using the distributional identity $\frac{1}{x \pm i \epsilon}=P\left(\frac{1}{x}\right) \mp i \pi \delta(x)$ and Equation (78). From this, one obtains an integral expression for the resolvent

$$
H(u)=-\frac{\alpha}{2}+\frac{1}{4}-\int_{a}^{b} d v \frac{v^{2}}{v^{2}-u^{2}} \sqrt{\frac{\left(b^{2}-u^{2}\right)\left(a^{2}-u^{2}\right)}{\left(b^{2}-v^{2}\right)\left(v^{2}-a^{2}\right)}},
$$

which in turn self-consistently yields the density

$$
\tilde{\rho}(u)=\frac{2}{\pi u} f_{a}^{b} d v \frac{v^{2}}{v^{2}-u^{2}} \sqrt{\frac{\left(b^{2}-u^{2}\right)\left(u^{2}-a^{2}\right)}{\left(b^{2}-v^{2}\right)\left(v^{2}-a^{2}\right)}},
$$


Finally, the interval boundaries $a$ and $b$ are implicitly determined through the normalization and positivity conditions on $\tilde{\rho}(u)$ (see $[16,8]$ ) by the relations

$$
\frac{1}{a}=4 K(q), \quad \frac{1}{b}=4 \sqrt{1-q} K(q), \quad q:=\frac{b^{2}-a^{2}}{b^{2}} .
$$

The resolvent and the density may be expressed in closed forms using the elliptic integral of the third kind ${ }^{8}$

$$
\begin{aligned}
H(u) & =-\frac{\alpha}{2}+\frac{1}{4}-\frac{\pi}{2} u-\frac{1}{4 b} \sqrt{\frac{a^{2}-u^{2}}{b^{2}-u^{2}}}\left[\frac{b^{2}}{a}-4 u^{2} \Pi\left(\frac{b^{2}-u^{2}}{b^{2}}, q\right)\right], \\
\tilde{\rho}(u) & =\frac{1}{2 \pi b u} \sqrt{\frac{u^{2}-a^{2}}{b^{2}-u^{2}}}\left[\frac{b^{2}}{a}-4 u^{2} \Pi\left(\frac{b^{2}-u^{2}}{b^{2}}, q\right)\right] .
\end{aligned}
$$

From this, it is straightforward to (finally) extract the energy eigenvalue $Q_{2}$ of the two cut solution in the parametric form

$$
E_{2}=\frac{1}{2 \pi^{2}} K(q)[2 E(q)-(2-q) K(q)]
$$

with

$$
\alpha=\frac{J_{2}}{J}=\frac{1}{2}-\frac{1}{2 \sqrt{1-q}} \frac{E(q)}{K(q)} .
$$

This final result for the one-loop gauge theory anomalous scaling dimension can now be compared to the folded string energies of Section 3.2, Equations $(34,35)$. They do not manifestly agree, however, if one relates the auxiliary parameters $q_{0}$ and $q$ (see [16]) through

$$
q_{0}=-\frac{(1-\sqrt{1-q})^{2}}{4 \sqrt{1-q}}
$$

one may show that

$$
K\left(q_{0}\right)=(1-q)^{1 / 4} K(q), \quad E\left(q_{0}\right)=\frac{1}{2}(1-q)^{-1 / 4} E(q)+\frac{1}{2}(1-q)^{1 / 4} K(q)
$$

using elliptic integral modular transformations. Using these relations, the gauge theory result (85, 86) may be transformed into the string result of $(34,35)$. Hence, the one-loop gauge theory scaling dimensions indeed agree with the string prediction!

The analysis of the circular string configuration goes along the same lines. Here, the Bethe roots turn out to condense on the imaginary axis. The root density is then symmetric along the imaginary axis, $\rho(u)=\rho(-u)$, and remains constant along a segment $[-c, c]$. For $u>c$ and $u<-c$ it falls off towards zero. We shall not go through the detailed construction of the density for this configuration but refer the reader to the original papers [22, 16], being best explained in [8]. The outcome of this analysis is again a perfect matching of the energy eigenvalue of the spin chain with the circular string energy of Equations $(42,43)$.

As a matter of fact, one can go beyond this and match all the higher charges of gauge and string theory, as was shown for the first time in [8], by using an approach based on the Bäcklund transform.

\subsection{Higher Loops in the $\mathrm{SU}(2)$ sector and discrepancies}

The connection to an integrable spin chain at one-loop raises the question whether integrability is merely an artifact of the one-loop approximation or a genuine property of planar $\mathcal{N}=4$ gauge theory. Remarkably, all present gauge theory data points towards the latter being the case.

\footnotetext{
${ }^{8}$ Our convention is $\Pi\left(m^{2}, q\right):=\int_{0}^{\pi / 2} \frac{d \phi}{\left(1-m^{2} \sin ^{2} \phi\right) \sqrt{1-q \sin ^{2} \phi}}$.
}

Living Reviews in Relativity

http://www. livingreviews.org//rr-2005-9 
Higher-loop contributions to the planar dilatation operator in the $\mathrm{SU}(2)$ subsector are by now firmly established for the two-loop [21] and three-loop level [12, 53]. In $s=1 / 2$ quantum spin chain language they take the explicit forms

$$
\begin{aligned}
\widehat{\mathcal{D}}_{2 \text {-loop }}= & \sum_{i=1}^{L}-\vec{\sigma}_{l} \cdot \vec{\sigma}_{l+2}+4 \vec{\sigma}_{l} \cdot \vec{\sigma}_{l+1}-3 \cdot \mathbf{1} \\
\widehat{\mathcal{D}}_{\text {3-loop }}= & \sum_{i=1}^{L}-\vec{\sigma}_{l} \cdot \vec{\sigma}_{l+3}+\left(\vec{\sigma}_{l} \cdot \vec{\sigma}_{l+2}\right)\left(\vec{\sigma}_{l+1} \cdot \vec{\sigma}_{l+3}\right)-\left(\vec{\sigma}_{l} \cdot \vec{\sigma}_{l+3}\right)\left(\vec{\sigma}_{l+1} \cdot \vec{\sigma}_{l+2}\right) \\
& \quad+10 \vec{\sigma}_{l} \cdot \vec{\sigma}_{l+2}-29 \vec{\sigma}_{l} \cdot \vec{\sigma}_{l+1}+20 \cdot \mathbf{1} .
\end{aligned}
$$

In general, the $k$-loop contribution to the dilatation operator involves interactions of $k+1$ neighboring spins, i.e. the full dilatation operator $\mathcal{D}=\sum_{k=1}^{\infty} \mathcal{D}_{k \text {-loop }}$ will correspond to a long-range interacting spin chain Hamiltonian. Note also the appearance of novel quartic spin interactions $\left(\vec{\sigma}_{i} \cdot \vec{\sigma}_{j}\right)\left(\vec{\sigma}_{k} \cdot \vec{\sigma}_{l}\right)$ at the three-loop level. Generically even higher interactions of the form $\left(\vec{\sigma}_{\bullet} \cdot \vec{\sigma}_{\bullet}\right)^{k}$ are expected at the $\left[\frac{k}{2}\right]+1$ loop levels.

Integrability remains stable up to the three-loop order and acts in a perturbative sense: The conserved charges of the Heisenberg $\mathrm{XXX}_{1 / 2}$ chain receive higher order corrections in $\lambda$ of the form $\mathcal{Q}_{k}=Q_{k}^{(1)}+\lambda Q_{k}^{(2)}+\lambda^{2} Q_{k}^{(3)}+\cdots$ as one would expect. The full charges $\mathcal{O}_{k}$ commute with each other $\left(\left[\mathcal{O}_{k}, \mathcal{O}_{l}\right]=0\right)$ which translates into commutation relations for the various loop contributions $\mathcal{O}_{k}^{(r)}$ upon expansion in $\lambda$, i.e.

$$
\begin{aligned}
{\left[Q_{k}^{(1)}, Q_{l}^{(1)}\right] } & =0 \\
{\left[Q_{k}^{(1)}, Q_{l}^{(2)}\right]+\left[Q_{k}^{(2)}, Q_{l}^{(1)}\right] } & =0 \\
{\left[Q_{k}^{(1)}, Q_{l}^{(3)}\right]+\left[Q_{k}^{(2)}, Q_{l}^{(2)}\right]+\left[Q_{k}^{(3)}, Q_{l}^{(1)}\right] } & =0,
\end{aligned}
$$$$
\cdots
$$

However, opposed to the situation for the Heisenberg chain [55], there does not yet exist an algebraic construction of the gauge theory charges at higher loops. Nevertheless, the first few $Q_{k}$ have been constructed manually to higher loop orders [14].

An additional key property of these higher-loop corrections is that they obey BMN scaling: The emergence of the effective loop-counting parameter $\lambda^{\prime}:=\lambda / J^{2}$ in the $J \rightarrow \infty$ limit leads to the scaling dimensions $\Delta \sim \sqrt{1+\lambda^{\prime} n^{2}}$ for two magnon states in quantitative agreement with plane-wave superstrings.

Motivated by these findings, Beisert, Dippel and Staudacher [15] turned the logic around and simply assumed integrability, BMN scaling and a Feynman diagrammatic origin of the $k$-loop $\mathrm{SU}(2)$ dilatation operator. Interestingly, these assumptions constrain the possible structures of the planar dilatation operator completely up to the five-loop level (and possibly beyond).

How can one now diagonalize the higher-loop corrected dilatation operator? For this, the ansatz for the Bethe wave-functions (57) needs to be adjusted in a perturbative sense in order to accommodate the long-range interactions, leading to a "perturbative asymptotic Bethe ansatz" for the two magnon wave-function [109]

$$
\begin{aligned}
\psi\left(x_{1}, x_{2}\right)=e^{i\left(p_{1} x_{1}+p_{2} x_{2}\right)} f\left(x_{2}-x_{1}, p_{1}, p_{2}\right) & \\
& +S\left(p_{2}, p_{1}\right) e^{i\left(p_{2} x_{1}+p_{1} x_{2}\right)} f\left(L-x_{2}+x_{1}, p_{1}, p_{2}\right) .
\end{aligned}
$$

Here, one needs to introduce a perturbative deformation of the S-matrix

$$
S\left(p_{1}, p_{2}\right)=S_{0}\left(p_{1}, p_{2}\right)+\sum_{n=1}^{\infty} \lambda^{n} S_{n}\left(p_{1}, p_{2}\right)
$$


which is determined by the eigenvalue problem. Moreover, suitable "fudge functions" enter the ansatz

$$
f\left(x, p_{1}, p_{2}\right)=1+\sum_{n=0}^{\infty} \lambda^{n+|x|} f_{n}\left(x, p_{1}, p_{2}\right) \stackrel{x \gg 1}{\longrightarrow} 1
$$

which account for a deformation of the plane-wave form of the eigenfunction when two magnons approach each other within the interaction range of the spin chain Hamiltonian ${ }^{9}$. By construction they are invisible in the asymptotic regime $x \gg 1$ (or rather $x$ larger than the highest loop order considered) of well separated magnons. The detailed form of these functions is completely irrelevant for the physical spectrum as a consequence of the factorized scattering property of the integrable system.

With this perturbative asymptotic Bethe ansatz (90), one shows that the form of the Bethe equations remains unchanged, i.e. the perturbative S-matrix (91) simply appears on the right hand side of the equations

$$
e^{i p_{k} L}=\prod_{i=1, i \neq k}^{M} S\left(p_{k}, p_{i}\right)
$$

and is determined by demanding $\psi\left(x_{1}, x_{2}\right)$ to be an eigenfunction of the dilatation operator, just as we did in Section 4.1. Based on the constructed five-loop form of the dilatation operator, the S-matrix is then determined up to $\mathcal{O}\left(\lambda^{4}\right)$. The obtained series in $\lambda$ turns out to be of a remarkably simple structure, which enabled the authors of [15] to conjecture an asymptotic all-loop expression for the perturbative S-matrix

$$
S\left(p_{1}, p_{2}\right)=\frac{\varphi\left(p_{1}\right)-\varphi\left(p_{2}\right)+i}{\varphi\left(p_{1}\right)-\varphi\left(p_{2}\right)-i} \quad \text { with } \quad \varphi(p)=\frac{1}{2} \cot \left(\frac{p}{2}\right) \sqrt{1+\lambda \sin ^{2}\left(\frac{p}{2}\right)}
$$

to be compared to the one-loop form of (59). The conjectured asymptotic all-loop form of the energy density generalizing the one-loop expression (65) reads ${ }^{10}$

$$
\lambda q_{2}(p)=\sqrt{1+8 \lambda \sin ^{2}\left(\frac{p}{2}\right)}-1
$$

with the total energy being given by $E_{2}=\sum_{i=1}^{M} q_{2}\left(p_{i}\right)$. Note that both expressions manifestly obey BMN scaling as the quasi-momenta scale like $p \sim L^{-1}$ in the thermodynamic limit as we discussed in Section 4.2. It is important to stress that these Bethe equations only make sense asymptotically: For a chain (or gauge theory operator) of length $L$ the Equations (94) and (95) yield a prediction for the energy (or scaling dimension) up to $L-1$ loops. This is the case, as the interaction range of the Hamiltonian will reach the length of the spin chain beyond this point, and the multi-magnon wave-functions of (90) can never enter the asymptotic regime. What happens beyond the $L$ loop level is still a mystery. At this point, the "wrapping" interactions start to set in: The interaction range of the spin chain Hamiltonian cannot spread any further and starts to "wrap" around the chain. In the dimensionally reduced model of plane-wave matrix theory [33, 49, 77, 50, 76, 78], these effects have been studied explicitly at the four-loop level [58] where the wrapping effects set in for the first time in the SU(2) subsector. No "natural" way of transforming the generic dilatation operator to the wrapping situation was found. Finally, let us restate that it has not been shown so far that a microscopic long-range spin chain Hamiltonian truly exists, which has a spectrum determined by the conjectured perturbative asymptotic Bethe equations (94) and (95) of Beisert, Dippel and Staudacher.

\footnotetext{
${ }^{9}$ We are actually using a slightly modified definition of these functions (compared to the one presented in [109]) which was considered in [58].

${ }^{10}$ The full conjecture for all the higher charge densities $q_{k}$ may be found in [15].
}

Living Reviews in Relativity

http: //www . livingreviews . org//rr-2005-9 
In any case, the proposed all-loop asymptotic Bethe equations $(94,95)$ may now be studied in the thermodynamic limit, just as we did above for the one-loop case. This was done in [107] and [15], and allows for a comparison to the results obtained in Section 3 for the energies of the spinning folded and closed string solutions. Recall that these yield predictions to all-loops in $\lambda^{\prime}$. While the two-loop gauge theory result is in perfect agreement, the three-loop scaling dimensions fail to match with the expected dual string theory result! This three-loop disagreement also arises in the comparison to the near plane-wave string spectrum computed in [46, 45, 44, 43], i.e. the first $1 / J$ corrections to the Penrose limit of $A d S_{5} \times S^{5}$ to the plane-wave background.

Does this mean that the AdS/CFT correspondence does not hold in its strong sense? While this logical possibility certainly exists, an alternative explanation is that one is dealing with an order-of-limits problem as pointed out initially in [15]. While in string theory one works in a limit of $\lambda \rightarrow \infty$ with $J^{2} / \lambda$ held fixed, in gauge theory one stays in the perturbative regime $\lambda \ll 1$ and thereafter takes the $J \rightarrow \infty$ limit, keeping only terms which scale as $\lambda / J^{2}$. These two limits need not commute. Most likely, the above-mentioned "wrapping" interactions must be included into the gauge theory constructions in order to match the string theory energies. On the other hand, the firm finite $L$ results at order $\lambda^{3}$ of the gauge theory are still free of "wrapping" interactions: These only start to set in at the four-loop level (in the considered minimal SU(2) subsector). Moreover, to what extent the integrability is preserved in the presence of these "wrapping" interactions is unclear at the moment. Certainly, the resolution of this discrepancy remains a pressing open problem in the field.

\subsection{Further developments}

The gauge theory analysis of the planar dilatation operator and its relation to integrable spin chain models has been extended in two directions: Larger sectors within $\mathcal{N}=4$ Super Yang-Mills and conformal deformations of the original theory.

In their seminal paper uncovering the integrable spin chain structure, Minahan and Zarembo [92] actually considered the full scalar sector of the gauge theory at one-loop order. This gives rise to an integrable $\mathrm{SO}(6)$ magnetic quantum spin chain of which the discussed $\mathrm{SU}(2)$ Heisenberg model arises in a subsector. This work was generalized in [11] to all local operators of the planar oneloop $\mathcal{N}=4$ theory, leading to an integrable super-spin chain with $\mathrm{SU}(2,2 \mid 4)$ symmetry discussed in [24]. The excitations of this super-spin chain consist of scalars, field strengths, fermions, and an arbitrary number of covariant derivatives of these three, leading to an infinite number of spin degrees of freedom on a single lattice site. The thermodynamic limit of this super-spin chain was later on constructed in larger supersymmetric subsectors in [105] and in [19] for the full system leading to spectral curves, which reproduce the results of the classical string at one-loop order.

The conjectured form of the asymptotic higher-loop Bethe ansatz for the PSU(2) subsector was generalized to the full theory recently in [25] in form of a long-range $\mathrm{SU}(2,2 \mid 4)$ Bethe ansatz. In this paper, the corresponding generalization for the quantum string Bethe equations, generalizing [6] relevant for the $\mathrm{SU}(2)$ sector, was also provided. A novel feature of leaving the minimal SU(2) sector at higher loop orders is that the spin chain begins to fluctuate in length [12]: The Hamiltonian (or dilatation operator) preserves the classical scaling dimensions but not the length of the chain, e.g. two fermions have the same classical scaling dimension as three scalars and can mix if they carry identical charges.

An alternative route for comparing string energies to gauge theory scaling dimensions lies in the coherent-state effective action approach pioneered by Kruczenski [79]. Here one establishes an effective action for the string whose center of mass moves along a big circle of the $S^{3}$ with large angular momentum in the "weak coupling" limit $\lambda / J^{2} \ll 1$. This action is then shown to agree with the long-wave length approximation of the gauge theory spin chain at one-loop. In this approach there is no need to compare explicit solutions any longer, however, considering higher- 
loop effects and fermions becomes more challenging in this language. For details see [80, 71, 111, 81, 70, 112, 29, 28] and also Tseytlin's review [116].

In view of the reviewed insights, an obvious next question to address is what can be said about the non-planar sector of the gauge theory dual to string interactions. The gauge theory dilatation operator is indeed known for the first two-loop orders in the $\mathrm{SU}(2)$ sector exactly, that is including all non-planar contributions. However, extracting physical data from it, such as amplitudes for the decay of single trace operators to double trace ones is nontrivial. This has been performed with great success for the case of two or three magnon excitations in the BMN limit being dual to the interacting plane-wave superstring (reviewed in [94]). Performing the same computation for a macroscopic number of magnons, thus describing the quantum decay of the discussed spinning strings, is complicated enormously by the complexity of the Bethe wave-function (62) for large $M$. The analysis on the string side, however, can be performed by considering a semiclassical decay process [96]. For a related discussion on the non-planar gauge theory aspects see [30].

An interesting toy model for $\mathcal{N}=4$ Super Yang-Mills is its dimensional reduction on a three sphere to a quantum mechanical system [76], which turns out to be the plane-wave matrix theory of $[33,49,77,50]$ related to M-theory on the plane-wave background. The Hamiltonian of this matrix quantum mechanics reduces to an integrable spin chain in the large $N$ limit, which - remarkably - is identical to the full $\mathcal{N}=4$ system up to the three-loop level in the overlapping $\mathrm{SU}(2 \mid 3)$ sector [78] (via a perturbative redefinition of the coupling constant). However, a recent four-loop study displays a breakdown of BMN scaling at this level of perturbation theory while integrability is stable [58]. What this finding implies for the full $\mathcal{N}=4$ model remains to be seen.

A recent line of research concerns the study of deformations of $\mathcal{N}=4$ Super Yang-Mills, which maintain the quantum conformal structure known as the Leigh-Strassler or $\beta$ deformations [82]. The one-loop dilatation operator was constructed in subsectors of the theory in [99, 32]. Moreover, the explicit construction of the supergravity background dual to the $\beta$ deformed gauge theory was achieved by Lunin and Maldacena [84]. Again, the classical bosonic string theory in this background is integrable and exhibits a Lax pair [65] yielding a string Bethe equation, which agrees with the thermodynamic limit of the one-loop Bethe equation for the gauge theory dilatation operator [59]. So, the complete discussion of this review lifts to the $\beta$ deformed case. As a matter of fact, even larger (three-parameter) families of generically non-supersymmetric deformations of $\mathcal{N}=4$ Super Yang-Mills have been considered with known supergravity duals [59]. The corresponding "twisted" gauge theory spin chain and Bethe ansatz was constructed in [23].

Open integrable spin chains have also appeared in the AdS/CFT setting where the boundaries of the spin chain correspond to fields in the fundamental representation; see [110, 47, 48, 51] for such constructions. In [34], open integrable spin chains emerged within subdeterminant operators in $\mathcal{N}=4$ Super Yang-Mills dual to so-called "giant gravitons".

First investigations on the role of integrability for the three-point functions in the gauge theory were performed in [93, 101, 3].

Finally, let us mention that integrable structures are known to also appear in QCD, such as in high-energy scattering processes and other instances [83, 56, 40, 26], see [27] for a recent review.

In conclusion, the emergence of integrable spin chains in the AdS/CFT correspondence has led to great insights into dynamical aspects of the duality and might hold the key to a complete determination of the spectrum of both theories. Recent developments point towards integrability being a generic property of conformal gauge theories in the planar limit not necessarily connected to supersymmetry. Finally, a great challenge for the future is to understand the integrable spin chain nature of the quantum string in $A d S_{5} \times S^{5}$ and related backgrounds.

Living Reviews in Relativity

http://www. livingreviews.org/lrr-2005-9 


\section{Acknowledgments}

I wish to thank Gleb Arutyunov, Niklas Beisert, Sergey Frolov, Matthias Staudacher, Arkady Tseytlin and Marija Zamaklar for helpful discussions and important comments on the manuscript. This review grew out of a lecture delivered at the Post-Strings 2004 Meeting at Durham. I thank the organizers of the meeting for hospitality and an inspiring workshop. 


\section{References}

[1] Aharony, O., Gubser, S.S., Maldacena, J.M., Ooguri, H., and Oz, Y., "Large N field theories, string theory and gravity", Phys. Rep., 323, 183-386, (2000). Related online version (cited on 13 July 2005):

http://arXiv.org/abs/hep-th/9905111. 1

[2] Alday, L.F., Arutyunov, G., and Tseytlin, A.A., "On integrability of classical superstrings in AdS(5) x S(5)", J. High Energy Phys., 2005(07), 002, (2005). Related online version (cited on 13 July 2005):

http://arXiv.org/abs/hep-th/0502240. 3.4

[3] Alday, L.F., David, J.R., Gava, E., and Narain, K.S., "Structure constants of planar N = 4 Yang Mills at one loop", J. High Energy Phys., 2005(09), 070, (2005). Related online version (cited on 13 July 2005):

http://arXiv.org/abs/hep-th/0502186. 4.4

[4] Arutyunov, G., and Frolov, S., "Integrable Hamiltonian for classical strings on $\operatorname{AdS}(5) \mathrm{x}$ S(5)", J. High Energy Phys., 2005(02), 059, (2005). Related online version (cited on 13 July 2005):

http://arXiv.org/abs/hep-th/0411089. 3.4

[5] Arutyunov, G., Frolov, S., Russo, J., and Tseytlin, A.A., "Spinning strings in AdS(5) x S(5) and integrable systems", Nucl. Phys. B, 671, 3-50, (2003). Related online version (cited on 13 July 2005):

http://arXiv.org/abs/hep-th/0307191. 3.4

[6] Arutyunov, G., Frolov, S., and Staudacher, M., "Bethe ansatz for quantum strings", J. High Energy Phys., 2004(10), 016, (2004). Related online version (cited on 13 July 2005): http://arXiv.org/abs/hep-th/0406256. 3.4, 4.4

[7] Arutyunov, G., Russo, J., and Tseytlin, A.A., "Spinning strings in $\operatorname{AdS}(5)$ x S(5): New integrable system relations", Phys. Rev. D, 69, 086009, (2004). Related online version (cited on 13 July 2005):

http://arXiv.org/abs/hep-th/0311004. 3.4

[8] Arutyunov, G., and Staudacher, M., "Matching higher conserved charges for strings and spins", J. High Energy Phys., 2004(03), 004, (2004). Related online version (cited on 13 July 2005):

http://arXiv.org/abs/hep-th/0310182. 4.2, 7, 7, 8

[9] Arutyunov, G., and Zamaklar, M., "Linking Baecklund and monodromy charges for strings on $\operatorname{AdS}(5)$ x S(5)", J. High Energy Phys., 2005(07), 026, (2005). Related online version (cited on 13 July 2005):

http://arXiv.org/abs/hep-th/0504144. 3.4

[10] Beisert, N., "BMN operators and superconformal symmetry", Nucl. Phys. B, 659, 79-118, (2003). Related online version (cited on 13 July 2005):

http://arXiv.org/abs/hep-th/0211032. 6

[11] Beisert, N., "The complete one-loop dilatation operator of $\mathrm{N}=4$ super Yang-Mills theory", Nucl. Phys. B, 676, 3-42, (2004). Related online version (cited on 13 July 2005):

http://arXiv.org/abs/hep-th/0307015. 4.4

Living Reviews in Relativity

http: //www . livingreviews . org/lrr-2005-9 
[12] Beisert, N., "The su(2-3) dynamic spin chain", Nucl. Phys. B, 682, 487-520, (2004). Related online version (cited on 13 July 2005):

http://arXiv.org/abs/hep-th/0310252. 1, 4.3, 4.4

[13] Beisert, N., "The dilatation operator of $\mathrm{N}=4$ super Yang-Mills theory and integrability", Phys. Rep., 405, 1-202, (2005). Related online version (cited on 13 July 2005):

http://arXiv.org/abs/hep-th/0407277. 2

[14] Beisert, N., "Spin chain for quantum strings", Fortschr. Phys., 53, 852-860, (2005). Related online version (cited on 13 July 2005):

http://arXiv.org/abs/hep-th/0409054. 3.4, 4.3

[15] Beisert, N., Dippel, V., and Staudacher, M., "A novel long range spin chain and planar N = 4 super Yang-Mills", J. High Energy Phys., 2004(07), 075, (2004). Related online version (cited on 13 July 2005):

http://arXiv.org/abs/hep-th/0405001. 4.3, 9, 10, 10

[16] Beisert, N., Frolov, S., Staudacher, M., and Tseytlin, A.A., "Precision spectroscopy of AdS/CFT", J. High Energy Phys., 2003(10), 037, (2003). Related online version (cited on 13 July 2005):

http://arXiv.org/abs/hep-th/0308117. 4.2, 7, 8, 8

[17] Beisert, N., Kazakov, V.A., and Sakai, K., "Algebraic curve for the $\mathrm{SO}(6)$ sector of AdS/CFT", (2004). URL (cited on 13 July 2005):

http://arXiv.org/abs/hep-th/0410253. 3.4

[18] Beisert, N., Kazakov, V.A., Sakai, K., and Zarembo, K., "The algebraic curve of classical superstrings on AdS(5) x S(5)", (2005). URL (cited on 13 July 2005):

http://arXiv.org/abs/hep-th/0502226. 3.4

[19] Beisert, N., Kazakov, V.A., Sakai, K., and Zarembo, K., "Complete spectrum of long operators in N = 4 SYM at one loop", J. High Energy Phys., 2005(07), 030, (2005). Related online version (cited on 13 July 2005):

http://arXiv.org/abs/hep-th/0503200. 4.4

[20] Beisert, N., Kristjansen, C., Plefka, J., and Staudacher, M., "BMN gauge theory as a quantum mechanical system", Phys. Lett. B, 558, 229-237, (2003). Related online version (cited on 13 July 2005):

http://arXiv.org/abs/hep-th/0212269. 4

[21] Beisert, N., Kristjansen, C., and Staudacher, M., "The dilatation operator of $\mathrm{N}=4$ super Yang-Mills theory", Nucl. Phys. B, 664, 131-184, (2003). Related online version (cited on 13 July 2005):

http://arXiv.org/abs/hep-th/0303060. 1, 2, 4, 4.3

[22] Beisert, N., Minahan, J.A., Staudacher, M., and Zarembo, K., "Stringing spins and spinning strings", J. High Energy Phys., 2003(09), 010, (2003). Related online version (cited on 13 July 2005):

http://arXiv.org/abs/hep-th/0306139. 4.2, 7, 7,8

[23] Beisert, N., and Roiban, R., "Beauty and the twist: The Bethe ansatz for twisted $\mathrm{N}=4$ SYM", J. High Energy Phys., 2005(08), 039, (2005). Related online version (cited on 13 July 2005):

http://arXiv.org/abs/hep-th/0505187. 4.4 
[24] Beisert, N., and Staudacher, M., "The N = 4 SYM integrable super spin chain", Nucl. Phys. $B$, 670, 439-463, (2003). Related online version (cited on 13 July 2005):

http://arXiv.org/abs/hep-th/0307042. 2, 4.4

[25] Beisert, N., and Staudacher, M., "Long-range PSU(2,2-4) Bethe ansaetze for gauge theory and strings", Nucl. Phys. B, 727, 1-62, (2005). Related online version (cited on 13 July 2005):

http://arXiv.org/abs/hep-th/0504190. 4.4

[26] Belitsky, A.V., "Renormalization of twist-three operators and integrable lattice models", Nucl. Phys. B, 547, 407, (2000). Related online version (cited on 03 November 2005):

http://arXiv.org/abs/hep-ph/9907420. 4.4

[27] Belitsky, A.V., Braun, V.M., Gorsky, A.S., and Korchemsky, G.P., "Integrability in QCD and beyond", Int. J. Mod. Phys. A, 19, 4715-4788, (2004). Related online version (cited on 13 July 2005):

http://arXiv.org/abs/hep-th/0407232. 4.4

[28] Bellucci, S., Casteill, P.Y., and Morales, F.F., "Superstring sigma models from spin chains: The SU(1,1-1) case", (2005). URL (cited on 03 November 2005):

http://arXiv.org/abs/hep-th/0503159. 4.4

[29] Bellucci, S., Casteill, P.Y., Morales, F.F., and Sochichiu, C., "Sl(2) spin chain and spinning strings on $\operatorname{AdS}(5)$ x S(5)", Nucl. Phys. B, 707, 303, (2005). Related online version (cited on 03 November 2005):

http://arXiv.org/abs/hep-th/0409086. 4.4

[30] Bellucci, S., Casteill, P.Y., Morales, J.F., and Sochichiu, C., "Spin bit models from nonplanar N = 4 SYM", Nucl. Phys. B, 699, 151-173, (2004). Related online version (cited on 13 July 2005):

http://arXiv.org/abs/hep-th/0404066. 4.4

[31] Bena, I., Polchinski, J., and Roiban, R., "Hidden symmetries of the AdS(5) x S(5) superstring", Phys. Rev. D, 69, 046002, (2004). Related online version (cited on 13 July 2005): http://arXiv.org/abs/hep-th/0305116. 2, 3.4

[32] Berenstein, D., and Cherkis, S.A., "Deformations of $\mathrm{N}=4 \mathrm{SYM}$ and integrable spin chain models", Nucl. Phys. B, 702, 49-85, (2004). Related online version (cited on 13 July 2005): http://arXiv.org/abs/hep-th/0405215. 4.4

[33] Berenstein, D., Maldacena, J.M., and Nastase, H., "Strings in flat space and pp waves from N = 4 super Yang Mills", J. High Energy Phys., 2002(04), 013, (2002). Related online version (cited on 13 July 2005):

http://arXiv.org/abs/hep-th/0202021. 1, 2.1, 6, 10, 4.4

[34] Berenstein, D., and Vazquez, S.E., "Integrable open spin chains from giant gravitons", J. High Energy Phys., 2005(06), 059, (2005). Related online version (cited on 13 July 2005): http://arXiv.org/abs/hep-th/0501078. 4.4

[35] Berkovits, N., "BRST cohomology and nonlocal conserved charges", J. High Energy Phys., 2005(02), 060, (2005). Related online version (cited on 13 July 2005): http://arXiv.org/abs/hep-th/0409159. 3.4 
[36] Berkovits, N., "Quantum consistency of the superstring in $\operatorname{AdS}(5)$ x $\mathrm{S}(5)$ background", $J$. High Energy Phys., 2005(03), 041, (2005). Related online version (cited on 13 July 2005): http://arXiv.org/abs/hep-th/0411170. 3.4

[37] Bethe, H., "On the theory of metals. 1. Eigenvalues and eigenfunctions for the linear atomic chain", Z. Phys., 71, 205-226, (1931). 4.1, 6

[38] Blau, M., Figueroa-O'Farrill, J., Hull, C., and Papadopoulos, G., "A new maximally supersymmetric background of IIB superstring theory", J. High Energy Phys., 2002(01), 047, (2002). Related online version (cited on 13 July 2005):

http://arXiv.org/abs/hep-th/0110242. 2.1

[39] Blau, M., Figueroa-O'Farrill, J., Hull, C., and Papadopoulos, G., "Penrose limits and maximal supersymmetry", Class. Quantum Grav., 19, L87-L95, (2002). Related online version (cited on 13 July 2005):

http://arXiv.org/abs/hep-th/0201081. 2.1

[40] Braun, V.M., Derkachov, S.E., and Manashov, A.N., "Integrability of three-particle evolution equations in QCD", Phys. Lett. B, 81, 2020, (1998). Related online version (cited on 03 November 2005):

http://arXiv.org/abs/hep-ph/9805225. 4.4

[41] Brink, L., Lindgren, O., and Nilsson, B.E.W., "The ultraviolet finiteness of the N=4 YangMills theory", Phys. Lett. B, 123, 323, (1983). 2.2

[42] Brink, L., Schwarz, J.H., and Scherk, J., "Supersymmetric Yang-Mills theories", Nucl. Phys. $B, \mathbf{1 2 1}, 77,(1977) . \quad 2.2$

[43] Callan Jr, C.G., Heckman, J., McLoughlin, T., and Swanson, I., "Lattice super Yang-Mills: A virial approach to operator dimensions", Nucl. Phys. B, 701, 180-206, (2004). Related online version (cited on 13 July 2005):

http://arXiv.org/abs/hep-th/0407096. 3.4, 10

[44] Callan Jr, C.G., McLoughlin, T., and Swanson, I., "Higher impurity AdS/CFT correspondence in the near-BMN limit", Nucl. Phys. B, 700, 271-312, (2004). Related online version (cited on 13 July 2005):

http://arXiv.org/abs/hep-th/0405153. 3.4, 10

[45] Callan Jr, C.G., McLoughlin, T., and Swanson, I., "Holography beyond the Penrose limit", Nucl. Phys. B, 694, 115-169, (2004). Related online version (cited on 13 July 2005):

http://arXiv.org/abs/hep-th/0404007. 3.4, 10

[46] Callan Jr, C.G. et al., "Quantizing string theory in AdS(5) x S(5): Beyond the pp-wave", Nucl. Phys. B, 673, 3-40, (2003). Related online version (cited on 13 July 2005):

http://arXiv.org/abs/hep-th/0307032. 3.4, 10

[47] Chen, B., Wang, X.-J., and Wu, Y.-S., "Integrable open spin chain in super Yang-Mills and the plane-wave / SYM duality", J. High Energy Phys., 2004(02), 029, (2004). Related online version (cited on 13 July 2005):

http://arXiv.org/abs/hep-th/0401016. 4.4

[48] Chen, B., Wang, X.-J., and Wu, Y.-S., "Open spin chain and open spinning string", Phys. Lett. B, 591, 170-180, (2004). Related online version (cited on 13 July 2005):

http://arXiv.org/abs/hep-th/0403004. 4.4 
[49] Dasgupta, K., Sheikh-Jabbari, M.M., and Van Raamsdonk, M., "Matrix perturbation theory for M-theory on a PP-wave", J. High Energy Phys., 2002(05), 056, (2002). Related online version (cited on 13 July 2005):

http://arXiv.org/abs/hep-th/0205185. 10, 4.4

[50] Dasgupta, K., Sheikh-Jabbari, M.M., and Van Raamsdonk, M., "Protected multiplets of M-theory on a plane wave", J. High Energy Phys., 2002(09), 021, (2002). Related online version (cited on 13 July 2005):

http://arXiv.org/abs/hep-th/0207050. 10, 4.4

[51] DeWolfe, O., and Mann, N., "Integrable open spin chains in defect conformal field theory", J. High Energy Phys., 2004(04), 035, (2004). Related online version (cited on 13 July 2005): http://arXiv.org/abs/hep-th/0401041. 4.4

[52] D'Hoker, E., and Freedman, D.Z., "Supersymmetric gauge theories and the AdS/CFT correspondence", (2002). URL (cited on 13 July 2005):

http://arXiv.org/abs/hep-th/0201253. 1

[53] Eden, B., Jarczak, C., and Sokatchev, E., "A three-loop test of the dilatation operator in N = 4 SYM", Nucl. Phys. B, 712, 157-195, (2005). Related online version (cited on 13 July 2005): http://arXiv.org/abs/hep-th/0409009. 1, 4.3

[54] Engquist, J., Minahan, J.A., and Zarembo, K., "Yang-Mills duals for semiclassical strings on AdS(5) x S(5)", J. High Energy Phys., 2003(11), 063, (2003). Related online version (cited on 13 July 2005):

http://arXiv.org/abs/hep-th/0310188. 4.2

[55] Faddeev, L.D., "How Algebraic Bethe Ansatz works for integrable model", (2005). URL (cited on 03 November 2005): http://arXiv.org/abs/hep-th/9605187. 6, 4.3

[56] Faddeev, L.D., and Korchemsky, G.P., "High-energy QCD as a completely integrable model", Phys. Lett. B, 342, 311, (1995). Related online version (cited on 03 November 2005): http://arXiv.org/abs/hep-th/9404173. 4.4

[57] Faddeev, L.D., and Reshetikhin, N.Y., "Integrability of the principal chiral field model in 1+1 dimension", Ann. Phys. (N.Y.), 167, 227-256, (1986). 3.4

[58] Fischbacher, T., Klose, T., and Plefka, J., "Planar plane-wave matrix theory at the four loop order: Integrability without BMN scaling", J. High Energy Phys., 2005(02), 039, (2005). Related online version (cited on 13 July 2005):

http://arXiv.org/abs/hep-th/0412331. 10, 9, 4.4

[59] Frolov, S., "Lax pair for strings in Lunin-Maldacena background", J. High Energy Phys., 2005(05), 069, (2005). Related online version (cited on 13 July 2005):

http://arXiv.org/abs/hep-th/0503201. 4.4

[60] Frolov, S., and Tseytlin, A.A., "Semiclassical quantization of rotating superstring in AdS(5) x S(5)", J. High Energy Phys., 2002(06), 007, (2002). Related online version (cited on 03 November 2005):

http://arXiv.org/abs/hep-th/0204226. 2 
[61] Frolov, S., and Tseytlin, A.A., "Multi-spin string solutions in AdS(5) x S(5)", Nucl. Phys. $B, \mathbf{6 6 8}, 77-110,(2003)$. Related online version (cited on 13 July 2005):

http://arXiv.org/abs/hep-th/0304255. 1, 3

[62] Frolov, S., and Tseytlin, A.A., "Quantizing three-spin string solution in $\operatorname{AdS}(5)$ x S(5)", J. High Energy Phys., 2003(07), 016, (2003). Related online version (cited on 03 November 2005):

http://arXiv.org/abs/hep-th/0306130. 1,3.4

[63] Frolov, S., and Tseytlin, A.A., "Rotating string solutions: AdS/CFT duality in nonsupersymmetric sectors", Phys. Lett. B, 570, 96-104, (2003). Related online version (cited on 13 July 2005):

http://arXiv.org/abs/hep-th/0306143. 3

[64] Frolov, S.A., Park, I.Y., and Tseytlin, A.A., "On one-loop correction to energy of spinning strings in S(5)", Phys. Rev. D, 71, 026006, (2005). Related online version (cited on 03 November 2005):

http://arXiv.org/abs/hep-th/0408187. 3.4

[65] Frolov, S.A., Roiban, R., and Tseytlin, A.A., "Gauge-string duality for superconformal deformations of $\mathrm{N}=4$ super Yang-Mills theory", (2005). URL (cited on 13 July 2005): http://arXiv.org/abs/hep-th/0503192. 4.4

[66] Gliozzi, F., Scherk, J., and Olive, D.I., "Supersymmetry, supergravity theories and the dual spinor model", Nucl. Phys. B, 122, 253-290, (1977). 2.2

[67] Grabowski, M.P., and Mathieu, P., "Quantum integrals of motion for the Heisenberg spin chain", (1994). URL (cited on 13 July 2005):

http://arXiv.org/abs/hep-th/9403149. 4

[68] Gubser, S.S., Klebanov, I.R., and Polyakov, A.M., "Gauge theory correlators from noncritical string theory", Phys. Lett. B, 428, 105-114, (1998). Related online version (cited on 13 July 2005):

http://arXiv.org/abs/hep-th/9802109. 3.4

[69] Gubser, S.S., Klebanov, I.R., and Polyakov, A.M., "A semi-classical limit of the gauge/string correspondence", Nucl. Phys. B, 636, 99, (2002). Related online version (cited on 03 November 2005):

http://arXiv.org/abs/hep-th/0204051. 2, 3.4

[70] Hernandez, R., and Lopez, E., "Spin chain sigma models with fermions", J. High Energy Phys., 2004(11), 079, (2004). Related online version (cited on 13 July 2005):

http://arXiv.org/abs/hep-th/0410022. 4.4

[71] Hernandez, R., and Lopez, E., "The SU(3) spin chain sigma model and string theory", $J$. High Energy Phys., 2004(04), 052, (2004). Related online version (cited on 03 November 2005):

http://arXiv.org/abs/hep-th/0403139. 4.4

[72] Howe, P.S., Stelle, K.S., and Townsend, P.K., "Miraculous ultraviolet cancellations in supersymmetry made manifest", Nucl. Phys. B, 236, 125, (1984). 2.2

[73] Karbach, M., and Muller, G., "Introduction to the Bethe ansatz I", Comput. Phys., 11, 36, (1997). Related online version (cited on 13 July 2005):

http://arXiv.org/abs/cond-mat/9809162. 6, 6 
[74] Kazakov, V.A., Marshakov, A., Minahan, J.A., and Zarembo, K., "Classical / quantum integrability in AdS/CFT", J. High Energy Phys., 2004(05), 024, (2004). Related online version (cited on 13 July 2005):

http://arXiv.org/abs/hep-th/0402207. 3.4

[75] Kazakov, V.A., and Zarembo, K., "Classical / quantum integrability in non-compact sector of AdS/CFT", J. High Energy Phys., 2004(10), 060, (2004). Related online version (cited on 13 July 2005):

http://arXiv.org/abs/hep-th/0410105. 3.4

[76] Kim, N., Klose, T., and Plefka, J., "Plane-wave matrix theory from N = 4 super Yang-Mills on R x S(3)", Nucl. Phys. B, 671, 359-382, (2003). Related online version (cited on 13 July 2005):

http://arXiv.org/abs/hep-th/0306054. 10, 4.4

[77] Kim, N., and Plefka, J., "On the spectrum of pp-wave matrix theory", Nucl. Phys. B, 643, 31-48, (2002). Related online version (cited on 13 July 2005):

http://arXiv.org/abs/hep-th/0207034. 10, 4.4

[78] Klose, T., and Plefka, J., "On the integrability of large N plane-wave matrix theory", Nucl. Phys. B, 679, 127-142, (2004). Related online version (cited on 13 July 2005):

http://arXiv.org/abs/hep-th/0310232. 10, 4.4

[79] Kruczenski, M., "Spin chains and string theory", Phys. Rev. Lett., 93, 161602, (2004). Related online version (cited on 13 July 2005):

http://arXiv.org/abs/hep-th/0311203. 4.4

[80] Kruczenski, M., Ryzhov, A.V., and Tseytlin, A.A., "Large spin limit of AdS(5) x S(5) string theory and low energy expansion of ferromagnetic spin chains", Nucl. Phys. B, 692, 3-49, (2004). Related online version (cited on 13 July 2005):

http://arXiv.org/abs/hep-th/0403120. 4.4

[81] Kruczenski, M., and Tseytlin, A.A., "Semiclassical relativistic strings in S(5) and long coherent operators in N = 4 SYM theory", J. High Energy Phys., 2004(09), 038, (2004). Related online version (cited on 13 July 2005):

http://arXiv.org/abs/hep-th/0406189. 4.4

[82] Leigh, R.G., and Strassler, M.J., "Exactly marginal operators and duality in four-dimensional $\mathrm{N}=1$ supersymmetric gauge theory", Nucl. Phys. B, 447, 95-136, (1995). Related online version (cited on 13 July 2005):

http://arXiv.org/abs/hep-th/9503121. 4.4

[83] Lipatov, L.N., "High-energy asymptotics of multicolor QCD and exactly solvable lattice models", (2005). URL (cited on 03 November 2005):

http://arXiv.org/abs/hep-th/9311037. 4.4

[84] Lunin, O., and Maldacena, J.M., "Deforming field theories with U(1) x U(1) global symmetry and their gravity duals", J. High Energy Phys., 2005(05), 033, (2005). Related online version (cited on 13 July 2005):

http://arXiv.org/abs/hep-th/0502086. 4.4

[85] Luscher, M., and Pohlmeyer, K., "Scattering of massless lumps and nonlocal charges in the two-dimensional classical nonlinear sigma model", Nucl. Phys. B, 137, 46, (1978). 3.4

Living Reviews in Relativity

http: //www . livingreviews . org/lrr-2005-9 
[86] Maldacena, J.M., "The large N limit of superconformal field theories and supergravity", Adv. Theor. Math. Phys., 2, 231-252, (1998). Related online version (cited on 13 July 2005): http://arXiv.org/abs/hep-th/9711200.

[87] Mandal, G., Suryanarayana, N.V., and Wadia, S.R., "Aspects of semiclassical strings in AdS(5)", Phys. Lett. B, 543, 81-88, (2002). Related online version (cited on 13 July 2005): http://arXiv.org/abs/hep-th/0206103. 3.4

[88] Metsaev, R.R., "Type IIB Green-Schwarz superstring in plane wave Ramond-Ramond background", Nucl. Phys. B, 625, 70-96, (2002). Related online version (cited on 13 July 2005): http://arXiv.org/abs/hep-th/0112044. 1, 2.1

[89] Metsaev, R.R., and Tseytlin, A.A., "Type IIB superstring action in AdS(5) x S(5) background", Nucl. Phys. B, 533, 109-126, (1998). Related online version (cited on 13 July 2005):

http://arXiv.org/abs/hep-th/9805028. 2

[90] Metsaev, R.R., and Tseytlin, A.A., "Exactly solvable model of superstring in plane wave Ramond-Ramond background", Phys. Rev. D, 65, 126004, (2002). Related online version (cited on 13 July 2005): http://arXiv.org/abs/hep-th/0202109. 1, 2.1

[91] Minahan, J.A., "Circular semiclassical string solutions on $\operatorname{AdS}(5)$ x $\mathrm{S}(5)$ ", Nucl. Phys. B, 648, 203, (2002). Related online version (cited on 03 November 2005):

http://arXiv.org/abs/hep-th/0209047. 3.4

[92] Minahan, J.A., and Zarembo, K., "The Bethe-ansatz for $\mathrm{N}=4$ super Yang-Mills", J. High Energy Phys., 2003(03), 013, (2003). Related online version (cited on 13 July 2005):

http://arXiv.org/abs/hep-th/0212208. 2, 4, 6, 4.4

[93] Okuyama, K., and Tseng, L.-S., "Three-point functions in N = 4 SYM theory at one-loop", J. High Energy Phys., 2004(08), 055, (2004). Related online version (cited on 03 November 2005):

http://arXiv.org/abs/hep-th/0404190. 4.4

[94] Pankiewicz, A., "Strings in plane wave backgrounds", Fortschr. Phys., 51, 1139-1203, (2003). Related online version (cited on 13 July 2005):

http://arXiv.org/abs/hep-th/0307027. 1, 2.1, 4.4

[95] Park, I.Y., Tirziu, A., and Tseytlin, A.A., "Spinning strings in AdS(5) x S(5): One-loop correction to energy in SL(2) sector", J. High Energy Phys., 2005(03), 013, (2005). Related online version (cited on 03 November 2005):

http://arXiv.org/abs/hep-th/0010104. 3.4

[96] Peeters, K., Plefka, J., and Zamaklar, M., "Splitting spinning strings in AdS/CFT", J. High Energy Phys., 2004(11), 054, (2004). Related online version (cited on 13 July 2005):

http://arXiv.org/abs/hep-th/0410275. 4.4

[97] Plefka, J., "Lectures on the plane-wave string / gauge theory duality", Fortschr. Phys., 52, 264-301, (2004). Related online version (cited on 13 July 2005):

http://arXiv.org/abs/hep-th/0307101. 1, 2.1, 4

[98] Pohlmeyer, K., "Integrable hamiltonian systems and interactions through quadratic constraints", Commun. Math. Phys., 46, 207-221, (1976). 3.4 
[99] Roiban, R., "On spin chains and field theories", J. High Energy Phys., 2004(09), 023, (2004). Related online version (cited on 13 July 2005):

http://arXiv.org/abs/hep-th/0312218. 4.4

[100] Roiban, R., and Siegel, W., "Superstrings on $\operatorname{AdS}(5)$ x S(5) supertwistor space", J. High Energy Phys., 2000(11), 024, (2000). Related online version (cited on 03 November 2005): http://arXiv.org/abs/hep-th/0010104. 2

[101] Roiban, R., and Volovich, A., "Yang-Mills correlation functions from integrable spin chains", J. High Energy Phys., 2004(09), 032, (2004). Related online version (cited on 13 July 2005): http://arXiv.org/abs/hep-th/0407140. 4.4

[102] Russo, J.G., "Anomalous dimensions in gauge theories from rotating strings in $\operatorname{AdS}(5) \mathrm{x}$ S(5)", J. High Energy Phys., 2002(06), 038, (2002). Related online version (cited on 03 November 2005):

http://arXiv.org/abs/hep-th/0205244. 3.4

[103] Russo, R., and Tanzini, A., "The duality between IIB string theory on pp-wave and N = 4 SYM: A status report", Class. Quantum Grav., 21, S1265-2196, (2004). Related online version (cited on 13 July 2005):

http://arXiv.org/abs/hep-th/0401155. 1, 2.1

[104] Sadri, D., and Sheikh-Jabbari, M.M., "The plane-wave / super Yang-Mills duality", Rev. Mod. Phys., 76, 853, (2004). Related online version (cited on 13 July 2005):

http://arXiv.org/abs/hep-th/0310119. 1, 2.1

[105] Schafer-Nameki, S., "The algebraic curve of 1-loop planar N =4 SYM", Nucl. Phys. B, 714, 3-29, (2005). Related online version (cited on 13 July 2005):

http://arXiv.org/abs/hep-th/0412254. 4.4

[106] Schafer-Nameki, S., Zamaklar, M., and Zarembo, K., "Quantum corrections to spinning strings in $\operatorname{AdS}(5)$ x S(5) and Bethe ansatz: A comparative study", (2005). URL (cited on 03 November 2005):

http://arXiv.org/abs/hep-th/0507189. 3.4

[107] Serban, D., and Staudacher, M., "Planar N = 4 gauge theory and the Inozemtsev long range spin chain", J. High Energy Phys., 2004(06), 001, (2004). Related online version (cited on 13 July 2005):

http://arXiv.org/abs/hep-th/0401057. 10

[108] Sohnius, M.F., and West, P.C., "Conformal invariance in N=4 supersymmetric Yang-Mills theory", Phys. Lett. B, 100, 245, (1981). 2.2

[109] Staudacher, M., "The factorized S-matrix of CFT/AdS", J. High Energy Phys., 2005(05), 054, (2005). Related online version (cited on 13 July 2005):

http://arXiv.org/abs/hep-th/0412188. 4.3, 9

[110] Stefanski Jr, B., "Open spinning strings", J. High Energy Phys., 2004(03), 057, (2004). Related online version (cited on 03 November 2005):

http://arXiv.org/abs/hep-th/0312091. 4.4

[111] Stefanski Jr, B., and Tseytlin, A.A., "Large spin limits of AdS/CFT and generalized LandauLifshitz equations", J. High Energy Phys., 2004(05), 042, (2004). Related online version (cited on 13 July 2005):

http://arXiv.org/abs/hep-th/0404133. 4.4

Living Reviews in Relativity

http: //www . livingreviews . org/lrr-2005-9 
[112] Stefanski Jr, B., and Tseytlin, A.A., "Super spin chain coherent state actions and AdS(5) x S(5) superstring", Nucl. Phys. B, 718, 83-112, (2005). Related online version (cited on 13 July 2005):

http://arXiv.org/abs/hep-th/0503185. 4.4

[113] Swanson, I., "Quantum string integrability and AdS/CFT", Nucl. Phys. B, 709, 443-464, (2005). Related online version (cited on 13 July 2005):

http://arXiv.org/abs/hep-th/0410282. 3.4

[114] Swanson, I., Superstring holography and integrability in $A d S(5) \times S(5)$, Ph.D. Thesis, (Caltech, Pasadena, U.S.A., 2005). Related online version (cited on 13 July 2005):

http://arXiv.org/abs/hep-th/0505028. 2

[115] 't Hooft, G., "A planar diagram theory for strong interactions", Nucl. Phys. B, 72, 461, (1974). 1

[116] Tseytlin, A.A., "Semiclassical strings and AdS/CFT", (2004). URL (cited on 13 July 2005): http://arXiv.org/abs/hep-th/0409296. 2, 4.4

[117] Tseytlin, A.A., "Spinning strings and AdS/CFT duality", in Shifman, M., Vainshtein, A., and Wheater, J., eds., From Fields to Strings: Circumnavigating Theoretical Physics (Ian Kogan Memorial Collection), (World Scientific, Singapore, 2005). Related online version (cited on 13 July 2005):

http://arXiv.org/abs/hep-th/0311139. 2

[118] Zakharov, V.E., and Mikhailov, A.V., "Relativistically Invariant Two-Dimensional Models in Field Theory Integrable by the Inverse Problem Technique", Sov. Phys. JETP, 47, 10171027, (1978). 3.4

[119] Zarembo, K., "Semiclassical integrability in AdS/CFT", C. R. Acad. Sci. Ser. IV, 5, 10811090, (2004). Related online version (cited on 13 July 2005):

http://arXiv.org/abs/hep-th/0411191. 2, 3.4 